\title{
Characterization of proanthocyanidin metabolism in pea (Pisum sativum) seeds
}

Kiva Ferraro ${ }^{1 \dagger}$, Alena L Jin ${ }^{2 \dagger}$, Trinh-Don Nguyen ${ }^{1}$, Dennis M Reinecke ${ }^{2}$, Jocelyn A Ozga ${ }^{2}$ and Dae-Kyun Ro ${ }^{1 *}$

\begin{abstract}
Background: Proanthocyanidins (PAs) accumulate in the seeds, fruits and leaves of various plant species including the seed coats of pea (Pisum sativum), an important food crop. PAs have been implicated in human health, but molecular and biochemical characterization of pea PA biosynthesis has not been established to date, and detailed pea PA chemical composition has not been extensively studied.

Results: PAs were localized to the ground parenchyma and epidermal cells of pea seed coats. Chemical analyses of PAs from seeds of three pea cultivars demonstrated cultivar variation in PA composition. 'Courier' and 'Solido' PAs were primarily prodelphinidin-types, whereas the PAs from 'LAN3017' were mainly the procyanidin-type. The mean degree of polymerization of 'LAN3017' PAs was also higher than those from 'Courier' and 'Solido'. Next-generation sequencing of 'Courier' seed coat cDNA produced a seed coat-specific transcriptome. Three cDNAs encoding anthocyanidin reductase (PSANR), leucoanthocyanidin reductase (PSLAR), and dihydroflavonol reductase (PSDFR) were isolated. PSANR and PSLAR transcripts were most abundant earlier in seed coat development. This was followed by maximum PA accumulation in the seed coat. Recombinant PsANR enzyme efficiently synthesized all three cis-flavan-3-ols (gallocatechin, catechin, and afzalechin) with satisfactory kinetic properties. The synthesis rate of trans-flavan-3-ol by co-incubation of PsLAR and PSDFR was comparable to cis-flavan-3-ol synthesis rate by PSANR. Despite the competent PSLAR activity in vitro, expression of PSLAR driven by the Arabidopsis ANR promoter in wild-type and anr knock-out Arabidopsis backgrounds did not result in PA synthesis.

Conclusion: Significant variation in seed coat PA composition was found within the pea cultivars, making pea an ideal system to explore PA biosynthesis. PsANR and PSLAR transcript profiles, PA localization, and PA accumulation patterns suggest that a pool of PA subunits are produced in specific seed coat cells early in development to be used as substrates for polymerization into PAs. Biochemically competent recombinant PsANR and PsLAR activities were consistent with the pea seed coat PA profile composed of both cis- and trans-flavan-3-ols. Since the expression of PSLAR in Arabidopsis did not alter the PA subunit profile (which is only comprised of cis-flavan-3-ols), it necessitates further investigation of in planta metabolic flux through PsLAR.
\end{abstract}

Keywords: Proanthocyanidin, Pea seeds, Pisum sativum, Anthocyanidin reductase, Flavan-3-ols, Flavonoid biosynthesis, Leucoanthocyanidin reductase

\section{Background}

Pisum sativum (pea) seeds are a rich source of minerals, proteins, starch and antioxidants. Dry pea seeds are widely used in agriculture as feed for livestock and are gaining interest as feed in aquaculture. Pea seeds, one of the oldest grain legumes consumed by humans, are also

\footnotetext{
* Correspondence: daekyun.ro@ucalgary.ca

${ }^{\dagger}$ Equal contributors

'Department of Biological Sciences, University of Calgary, 2500 University Dr. NW, Calgary, Alberta, Canada

Full list of author information is available at the end of the article
}

gaining wide recognition as a healthy food ingredient in the human diet due to the low glycemic index of the starches [1].

Flavonoids are of particular interest due to their strong antioxidant properties. Proanthocyanidins (PAs; Figure 1), also known as condensed tannins, are a subclass of flavonoids that accumulate in seed coats of a number of plant species including pea, and are thought to function as protective agents against biotic and abiotic stresses [2]. Historically, PAs were considered as anti-nutritional compounds in pulse nutritional studies because they

\section{Ciomed Central}

(c) 2014 Ferraro et al.; licensee BioMed Central Ltd. This is an Open Access article distributed under the terms of the Creative Commons Attribution License (http://creativecommons.org/licenses/by/4.0), which permits unrestricted use, distribution, and reproduction in any medium, provided the original work is properly credited. The Creative Commons Public Domain Dedication waiver (http://creativecommons.org/publicdomain/zero/1.0/) applies to the data made available in this article, unless otherwise stated. 


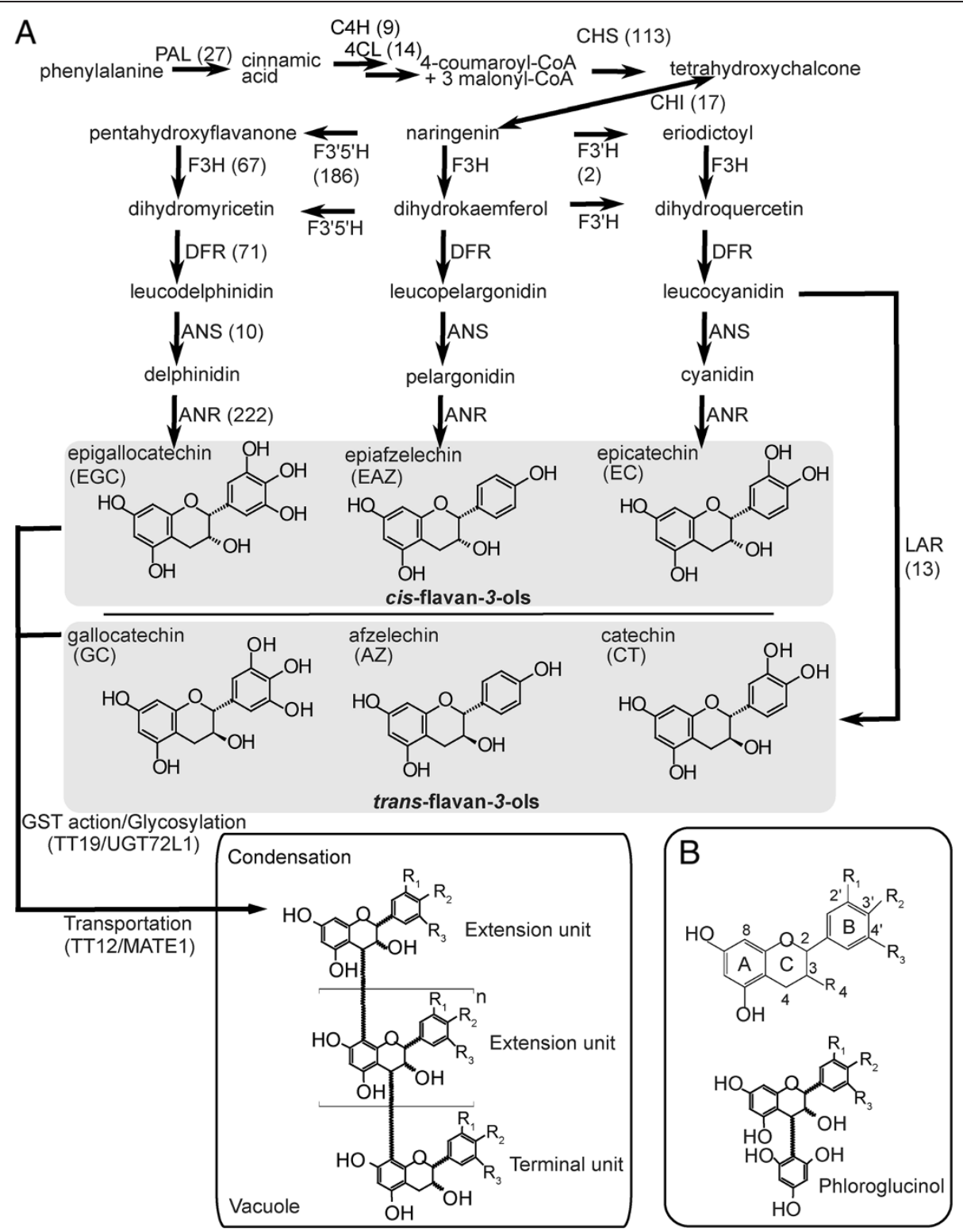

Figure 1 Proanthocyanidin biosynthetic pathway with transcript levels of each biosynthetic gene estimated by 454 read numbers, and structures of proanthocyanidins and their derivatized products. A) Proanthocyanidin biosynthetic pathway. PAL, phenylalanine ammonia lyase; C4H, cinnamate 4-hydroxylase; $4 \mathrm{CL}$, 4-coumarate:CoA ligase; CHS, chalcone synthase; $\mathrm{CHI}$, chalcone isomerase; F3'5'H, flavonoid 3'5'-hydroxylase; F3'H, flavonoid 3'-hydroxylase; F3H, flavanone 3-hydroxylase; DFR, dihydroflavonal 4-reductase; ANS, anthocyanidin synthase; ANR, anthocyanidin reductase; LAR, leucoanthocyanidin reductase. Values in brackets indicate the read numbers from 454-pyrosequencing. B) C4-C8 linkage in PAphloroglucinol adduct structures.

can precipitate proteins and reduce bioavailability of some minerals. However, recent research suggests that PAs have considerable potential for use as a novel therapy or treatment for a range of human health conditions, including cardiovascular disease, cancer establishment and progression, and bacterial infections [3]. The use of PAs as a plant-based health-beneficial component in the human diet has led to renewed interest in this class of flavonoids in food crops $[4,5]$. Specifically, studies indicate that PA polymer length is inversely related to bioavailability in humans [6]. Therefore, identification of variation in PA composition and length within Pisum sativum, as well as the mechanisms responsible for this variation would be a great benefit for breeding new cultivars with additional health beneficial properties.

PAs are derived from the flavonoid branch of the phenylpropanoid pathway (Figure 1). Chemical diversity can be introduced early in the pathway by regio-selective cytochrome P450 enzymes, F3' $\mathrm{H}$ and F3'5' $\mathrm{H}$ (Figure 1, see legend for full names), which hydroxylate 3 ' - or 
$3^{\prime}, 5^{\prime}$-positions of naringenin B-ring $[7,8]$. Two consecutive reactions by $\mathrm{F} 3 \mathrm{H}$ [9] and DFR then synthesize colorless flavan-3,4-diols (leucoanthocyanidins) [10], which are further converted to (-)-cis-flavan-3-ols through the sequential reactions of anthocyanidin synthase (ANS) [11] and anthocyanidin reductase (ANR) [12] or to (+)-trans-flavan-3-ols by leucoanthocyanidin reductase (LAR) [13].

Biosynthesis of these flavan-3-ol monomers is believed to occur on the cytosolic surface of the endoplasmic reticulum, yet PAs themselves accumulate in the vacuole $[14,15]$. Two multi-drug and toxic compound extrusion (MATE) transporters, TT12 and MATE1, characterized from Arabidopsis thaliana and Medicago truncatula, respectively, are able to transport epicatechin-3-O-glucoside (glycosylated cis-flavan-3-ol) across the tonoplastic membrane, but they were not able to transport aglycones (i.e., cyanidin and epicatechin) $[16,17]$. Therefore, glycosylation of flavan-3-ols appears to be necessary for the MATE-mediated transport, which is further supported by the recent discovery of an epicatechin-specific glycosyltransferase from $M$. truncatula [18]. Mechanistically, the MATE transporters are flavonoid $\mathrm{H}^{+}$-anti-porters, and the proton gradient required for this $\mathrm{H}^{+}$-anti-porter is believed to be generated by AHA10 (a $\mathrm{H}^{+}$-ATPase) on the tonoplast membrane [19].

In contrast to the transporter-mediated delivery of PA monomers, vesicle-mediated transport has also been proposed in planta. Arabidopsis mutant tt19, which encodes a glutathione-S-transferase-like protein, accumulates PA derivatives including flavan-3-ols in small vacuole-like structures [20]. TT19 may itself bind flavonoids to protect them from oxidation in the cytosol rather than conjugate glutathione to the flavan-3-ols [21]. A Golgi-independent vesicle-mediated trafficking pathway has also been proposed for anthocyanins, a group of pigments closely related to flavan-3-ols [22]. Recently, vesicles containing PA were identified and named as tannosome from grape (Vitis vinifera) and several other vascular plants [23]. This result also supports the implication of vesicle-mediated trafficking, but the vesicles appear to be derived from chloroplasts, which is in contrast to the ER/cytosolic biosynthesis of PA and hence requires further investigation.

PA polymers consist of flavan-3-ol aglycone subunits, suggesting a $\beta$-glucosidase within the vacuole may be required. Alternatively, deglycosylation may be coupled with condensation, which itself remains unknown. PA polymer length, composition of subunits, and $\mathrm{C}-\mathrm{C}$ bond stereochemistry varies between plant species, suggesting enzymatic control of condensation [3]. Laccases and peroxidases have been considered as potential condensing enzymes, although to date no PA condensing enzyme has been identified. One candidate, TT10, a putative laccase-like polyphenol oxidase, was proposed, but this enzyme appears to function in the apoplastic space where it converts colourless extractable PAs into their brown non-extractable oxidized form [24]. However, TT10 recombinant enzyme can oxidize epicatechin (EC), resulting in the formation of oligomers, although the resulting in vitro interflavan linkages are not naturally occurring [24]. It is possible that a protein partner, such as the dirigent protein involved in lignin coupling [25], is necessary for proper PA oligomerization, but non-enzymatic polymerization has not yet been ruled out [3].

Much of the research on seed coat-derived PAs has been conducted using the non-crop species Arabidopsis and M. truncatula. However, both of these species produce PA polymers composed almost exclusively of the cis-flavan-3-ol, epicatechin (Figure 1) [14,26]. Pea offers unique advantages to study PA biosynthesis. Pea seeds are substantially larger than those of Arabidopsis and $M$. truncatula, allowing for ready isolation of the seed coat tissue, the primary site of PA accumulation [27]. Also, a long history of agricultural breeding of pea has produced a wide variety of pea cultivars. Thousands of accessions of pea (Pisum sativum) exist around the world, providing both a rich source of genetic diversity and nutritional variation [28]. It is likely that variations in PA composition and polymer length exist in pea, and this could provide valuable resources to improve desirable PAs by breeding or biotechnological means. Despite the importance of pea as a crop and the possible value in understanding pea PA metabolism, comprehensive chemical and biochemical studies of PAs in pea have not been achieved to date. As the first step to advance the knowledge of PA biosynthesis in pea, we histologically localized PAs, determined PA accumulation, and chemically characterized the PAs of three PA-accumulating cultivars within the pea seed coat over development. The transcript abundance of two key PA branch point genes, PsANR and PSLAR, were profiled over development, and the enzymes they encode were biochemically characterized. Using these data, we developed a working hypothesis of PA biosynthesis in pea seed coat tissue.

\section{Results}

Localization of PAs in developing 'Courier' pea seed coats PAs were localized in the pea seed coats of 'Courier' (Figure 2) over development using a $p$-dimethylaminocinnamaldehyde (DMACA) staining method [29]. PAs mainly accumulated intracellularly (likely the vacuole) in the cells of the epidermal and ground parenchyma layers of the seed coat throughout development (Figure 2). As the seed matured, the cells of the epidermal layer of the seed coat sclerified, and the intercellular space and vacuolar size decreased. As a result, the vacuolar-localized PAs are visualized in the inner side of the epidermal layer. Also note that the inner seed coat cell layers are progressively 

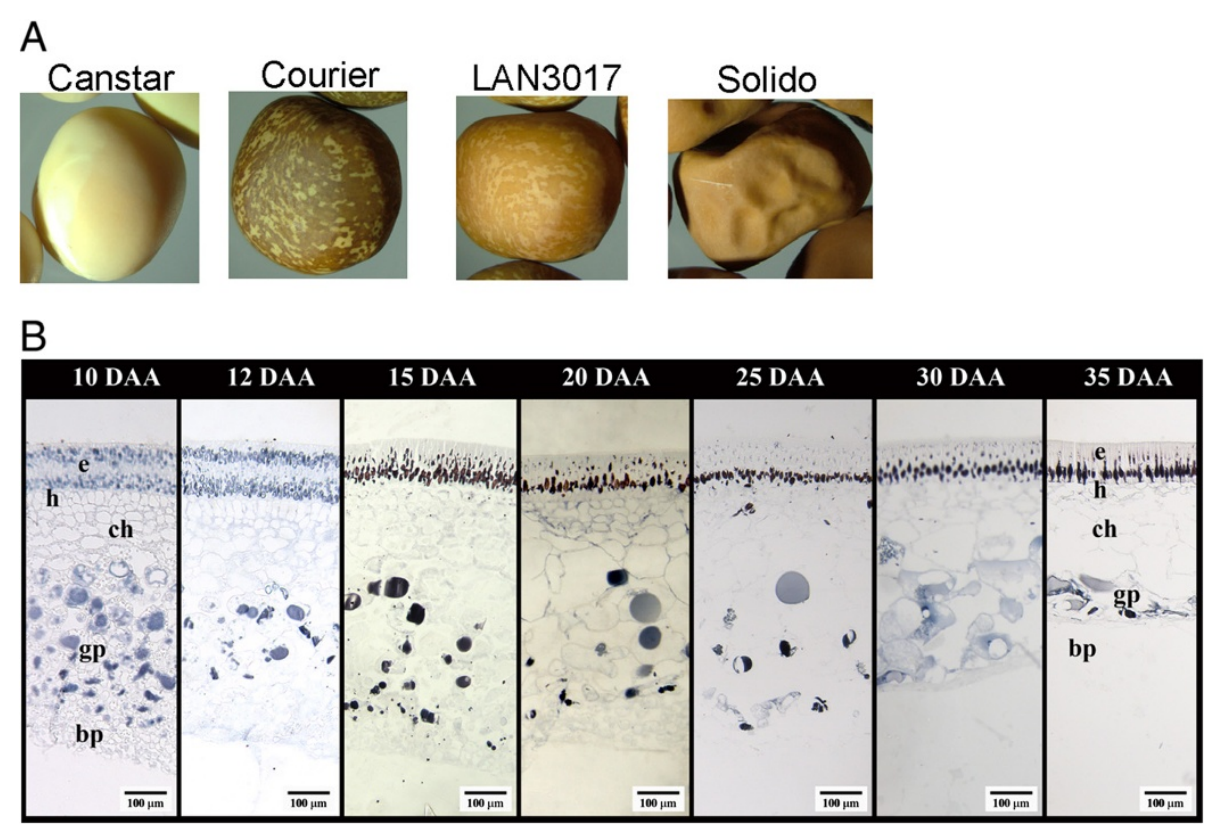

Figure 2 Pea seeds and PA localization in developing pea seed coat. A) Representative images of pea seeds from each cultivar. B) Cotyledon mid-region cross sections of 'Courier' pea seed coats. e, epidermal layer; h, hypodermal layer; ch, chlorenchyma layer; gp, ground parenchyma layer; bp, branched parenchyma layer. DAA: days after anthesis.

crushed by the expanding embryo as the seed develops (after 15 DAA; Figure 2).

\section{Proanthocyanidin profile of Pisum sativum cultivars}

The PA content and subunit composition of the seed coats from three PA-accumulating pea cultivars (Courier, LAN3017, and Solido) and a cultivar containing minimal PAs (Canstar) were determined by acid-catalyzed cleavage followed by phloroglucinol derivatization (phloroglucinolysis) (Table 1 and Figure 3) [30]. This method allows the determination of PA subunit composition and concentration by the comparison of the retention properties of reaction products with those of flavan-3-ol standards and other well characterized PA phloroglucinol reaction products. Flavan-3-ol PA extension units form phloroglucinol adducts at their $\mathrm{C} 4$ position while terminal flavan-3-ol units are released as flavan-3-ol monomers (Figure 1), the ratio of which allows determination of the mean degree of polymerization (mDP).

No PA subunits were detected in the RP-HPLC chromatography of 'Canstar' seed extracts ('Canstar' has clearcoloured seed coats, and the yellowish colouration of the seed is from the cotyledons; Figure 2A and 3). PA subunits were detected in the seed extracts of cultivars 'Courier', 'Solido', and 'Lan3017', that have brown or brown-speckled seed coats (Table 1; Figures 2A and 3). In the seeds of pea cultivars 'Courier' and 'Solido', similar PA flavan-3-ol extension and terminal unit profiles were detected (Table 1). The PA flavan-3-ol extension units were nearly exclusively prodelphinidin $\left(2^{\prime}, 3^{\prime}, 4^{\prime}\right.$-hydroxylated flavan-3-ols), where
Table 1 PA chemical analyses of 'Courier', 'Solido', and 'LAN3017' pea seeds and seed coats

PA analysis using phloroglucinolysis and RP-HPLC-DAD in mature pea seeds

\begin{tabular}{lllll}
\hline Peak ID & Compound & 'Courier' & 'Solido' & 'LAN3017' \\
\hline 3 & GC-P & $29.23 \pm 0.73^{\mathrm{a}}$ & $28.99 \pm 0.88$ & $1.36 \pm 0.02$ \\
4 & EGC-P & $55.38 \pm 1.05$ & $51.16 \pm 1.27$ & $0.79 \pm 0.05$ \\
5 & GC & $9.88 \pm 0.29$ & $10.53 \pm 0.14$ & $\mathrm{nd}^{\mathrm{b}}$ \\
6 & CT-P isomer & $\mathrm{nd}$ & $\mathrm{nd}$ & $4.98 \pm 0.00$ \\
7 & CT-P & nd & $0.22 \pm 0.01$ & $21.41 \pm 0.01$ \\
8 & EC-P & $0.37 \pm 0.01$ & $0.60 \pm 0.02$ & $65.37 \pm 0.03$ \\
9 & EGC & $5.13 \pm 0.04$ & $8.24 \pm 0.21$ & $\mathrm{nd}$ \\
10 & CT & $\mathrm{nd}$ & $\mathrm{nd}$ & $0.94 \pm 0.00$ \\
11 & EC & $\mathrm{nd}$ & $0.27 \pm 0.02$ & $5.15 \pm 0.02$ \\
mDP & & $6.7 \pm 0.2$ & $5.3 \pm 0.1$ & $16.4 \pm 0.0$ \\
Conversion yield & $83.9 \pm 1.6$ & $78.3 \pm 4.9$ & $59.1 \pm 0.9$ \\
Total seed PA & $416.0 \pm 7.7$ & $264.1 \pm 14.6$ & $96.7 \pm 13.2$
\end{tabular}

Butanol-HCl quantification of PA content from pea seed coats

Total seed coat PA (\%) $)^{\mathrm{e}} \quad 4.57 \pm 0.03 \quad 4.51 \pm 0.09 \quad 5.10 \pm 0.07$

${ }^{a}$ Molar $\% \pm S E(n=2) ;{ }^{b} n d$, not detected; ${ }^{c}$ Yield of PA extract calculated.

${ }^{\mathrm{d}}$ Total seed PA content based on characterized PA subunits, expressed as $\mathrm{mg} /$ $100 \mathrm{~g}$ dry weight of whole seeds. GC-P, gallocatechin- $(4 \alpha \rightarrow 2)$-phloroglucinol; GC, gallocatechin; EGC-P, epigallocatechin- $(4 \beta \rightarrow 2)$-phloroglucinol; EGC, epigallocatechin; CT-P, catechin- $(4 a \rightarrow 2)$-phloroglucinol; CT, catechin; EC-P, epicatechin- $(4 \beta \rightarrow 2)$-phloroglucinol; EC, epicatechin.

${ }^{\mathrm{e}}$ Total seed coat PA content expressed as $\%=\mathrm{mg} / 100 \mathrm{mg}$ dry weight of seed coat sample using $80 \%$ methanol extraction. Proanthocyanidin extract from 'CDC Acer' pea seed coats purified as described by Jin et al. [41] was used as a standard for the butanol- $\mathrm{HCl}$ assay. Data are means \pm SE $(n=3)$. 


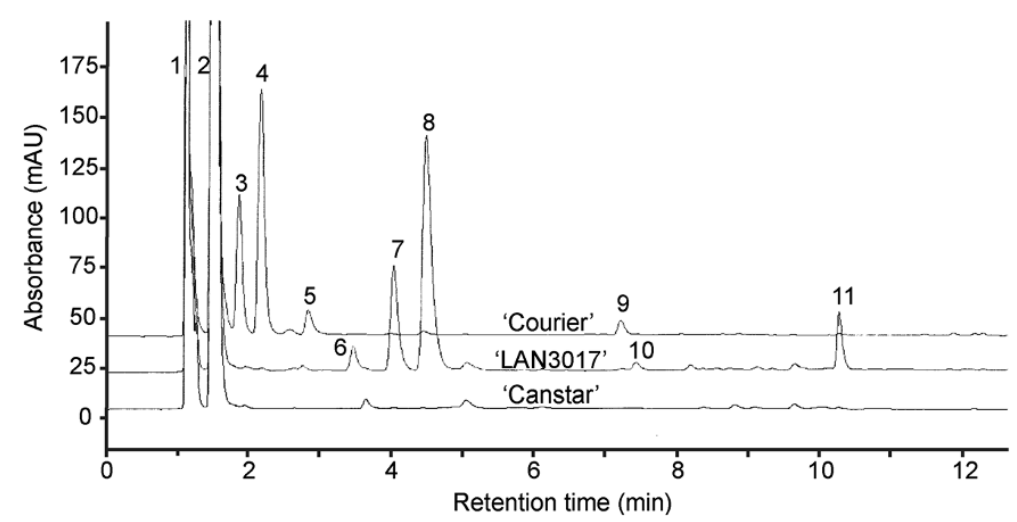

Figure 3 HPLC chromatograms of the phloroglucinol acid hydrolysis products from pea seeds of 'Courier', 'LAN3017', and 'Canstar'. 1. L-Ascorbic acid; 2. Phloroglucinol; 3. Gallocatechin-(4a-2)-phloroglucinol; 4. Epigallocatechin-(4ß-2)-phloroglucinol; 5. Gallocatechin; 6. Putative

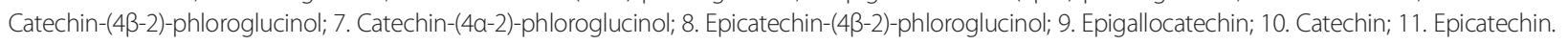

epigallocatechin (EGC; peak 4, Figure 3; Table 1) was the most abundant flavan-3-ol extension subunit followed by gallocatechin (GC; peak 3, Figure 3; Table 1). The PA terminal subunits of these pea cultivars mainly consisted of GC (peak 5) and EGC (peak 9, Figure 3; Table 1). A minimal amount of epicatechin (EC) also occurred in the PA extension subunits (peak 8) in these two cultivars, and in the terminal subunits (peak 11) of 'Solido' (Figure 3; Table 1). On the other hand, the PA flavan-3-ol extension and terminal subunit profile of 'LAN3017' seeds was markedly different from those of 'Solido' and 'Courier' (Figure 3; Table 1). 'LAN3017' contained nearly exclusively procyanidin $\left(2^{\prime}, 3^{\prime}\right.$-hydroxylated flavan-3-ols) moieties in the PA polymers, with the majority of the PA extension subunits consisting of EC (peak 8, Figure 3) followed by catechin (CT; peak 7, Figure 3; Table 1).

The mDP of the PA polymers was similar in 'Courier' and 'Solido' at 5-7 subunits in length. However, the PA $\mathrm{mDP}$ was 2 to 3 times greater in 'LAN3017' than that in the other pea cultivars (Table 1). The PA extension and terminal subunits in the PA-containing pea cultivars are assumed to be linked in a B-type configuration (C4-C8 or C4-C6) (Figure 1A; C4-C8), as the PA interflavonoid bonds were readily cleaved under the acidic conditions. The identities of the PA subunits detected in the HPLC analysis were further substantiated by LC-MS/MS (Additional file 1: Table S1).

Similar PA levels were found among the PA-containing pea cultivars when the total extractable PA content of the seed coat was estimated using the butanol- $\mathrm{HCl}$ method (Table 1). The total extractable PA yield from whole seed extracts was also calculated using the PA extract yield values and the conversion yield of PAs to known subunits with data from the phloroglucinolysis method (Table 1) [31]. The lower PA content values obtained in the whole seed extracts compared to the seed coat extracts are the result of: 1) PA localization in the seed coat and not the embryo of the seeds for all cultivars, and 2) a larger ratio of embryo to seed coat tissue in the seeds of 'Solido', and decreased solubility of the longer PA polymers of 'LAN3017' in the extraction solvent used in the phloroglucinolysis procedure compared to the shorter PA polymers present in 'Courier' and 'Solido'. Therefore, the total extractable PA content of the seed coat as estimated using the butanol- $\mathrm{HCl}$ assay is the method of choice for determining PA content difference among these cultivars.

To further understand PA accumulation in the pea seed coat, the content and composition of 'Courier' extractable PAs over development were examined. The molar percent of GC in the extension units increased as seed development progressed, while a small decrease in EGC occurred (Table 2). The mDP of PAs from young seed coats at 12 days after anthesis (DAA) was less than five, then it increased slightly (about one subunit in length) by 15 DAA and it remained at this level until 30 DAA (Figure 4A). At seed maturity, the $\mathrm{mDP}$ increased to approximately seven (Table 1). The extractable seed coat PA content increased during development, reaching a maximum level at 20 DAA (Figure 4A). After 20 DAA, the extractable PA content steadily decreased until seed maturation.

\section{Cloning and characterization $P$. sativum ANR}

Pea seed coat PA subunits consisted of a high quantity of trans-flavan-3-ols (GC and $\mathrm{CT}$ ) in addition to common cis-flavan-3-ols (EGC and EC; Table 1). In contrast, the PAs of the closely related legume species Medicago truncatula and the model plant Arabidopsis thaliana are reported to not contain trans-flavan-3-ol subunits. These results imply that both LAR and ANR, the key enzymes responsible for the biosynthesis of PA precursors, are highly active in the pea seed coat PA biosynthesis pathway (Figure 1). No biochemical studies of these two key branch enzymes have been conducted in the crop species pea, 
Table 2 PA profiles in developing seed coats of 'Courier'

\begin{tabular}{lllllll}
\hline & GC-P & EGC-P & EC-P & GC & EGC & EC \\
\hline 12 DAA & $20.5 \pm 1.9^{\text {b }}$ & $55.8 \pm 1.9$ & $0.42 \pm 0.02$ & $17.3 \pm 0.4$ & $5.65 \pm 0.26$ & $0.38 \pm 0.03$ \\
15 DAA & $24.8 \pm 0.7$ & $55.2 \pm 0.1$ & $0.38 \pm 0.03$ & $15.0 \pm 0.3$ & $4.35 \pm 0.36$ & $0.32 \pm 0.10$ \\
20 DAA & $27.5 \pm 0.6$ & $52.9 \pm 0.7$ & $0.36 \pm 0.04$ & $14.4 \pm 0.7$ & $4.47 \pm 0.19$ & $0.29 \pm 0.07$ \\
25 DAA & $29.2 \pm 0.6$ & $51.6 \pm 1.0$ & $0.22 \pm 0.19$ & $14.3 \pm 0.4$ & $4.51 \pm 0.16$ & $0.21 \pm 0.01$ \\
30 DAA & $30.8 \pm 1.7$ & $49.8 \pm 2.2$ & $0.31 \pm 0.04$ & $14.1 \pm 0.4$ & $4.76 \pm 0.21$ & $0.19 \pm 0.05$ \\
\hline
\end{tabular}

${ }^{\mathrm{a}} \mathrm{DAA}$, days after anthesis; ${ }^{\mathrm{b}}$ Molar $\% \pm \mathrm{SE}(\mathrm{n}=3)$.

GC-P, gallocatechin- $(4 a \rightarrow 2)$-phloroglucinol; EGC-P, epigallocatechin- $(4 \beta \rightarrow 2)$-phloroglucinol; EC-P, epicatechin-(4 $\rightarrow \rightarrow 2)$-phloroglucinol; GC, gallocatechin; EGC, epigallocatechin; EC, epicatechin.

PA content was determined using the phloroglucinolysis and RP-HPLC-DAD analysis method.

and thus we pursued thorough biochemical studies of ANR and LAR.

'Courier' was chosen as the source of a PSANR clone as this cultivar displayed high seed coat PA accumulation as well as significant quantities of cis-flavan-3-ol PA subunits (Table 1). A full-length PSANR clone was retrieved from 'Courier' seed coat cDNA using degenerate PCR, followed by rapid amplification of cDNA ends (5'-, 3'RACE). PsANR encodes a 1,017-bp ORF and shares $84 \%$ and $60 \%$ amino acid identity with $M$. truncatula ANR and Arabidopsis ANR, respectively. PsANR is highly conserved among 'Courier', 'LAN3017' and 'Solido', differing by only a single amino acid in 'LAN3017' (position 28, Gln to Glu) and in 'Solido' (position 327, Ile to Val).

To examine the catalytic activity of PsANR, PSANR was expressed as an $\mathrm{N}$-terminal six-histidine tagged recombinant protein and purified using a Ni-NTA column. Based on the pea PA subunit composition data, the primary in planta substrate for 'Courier' PsANR is expected to be the $2^{\prime}, 3^{\prime}, 4^{\prime}$-hydroxylated anthocyanidin, delphinidin (Figure 1). Therefore, delphinidin as well as two related compounds, $2^{\prime}, 3^{\prime}$-hydroxylated cyanidin and $3^{\prime}$-hydroxylated pelargonidin, were assessed as substrates for recombinant PsANR (Figure 5). When the PsANR enzymatic products were analyzed by LC-MS/MS, they showed identical co-chromatographic and MS/MS patterns with the corresponding authentic cis-flavan-3-ol standards, EGC, EC and EAZ (Figure 5 and Additional file 2: Figure S1). No flavan-3-ol product was detected when NADPH was omitted or if the protein was boiled prior to the assay (data not shown). These results showed that all three compounds can be efficiently used as substrates to produce cis-flavan-3-ols, and that nonenzymatic conversion of cis-flavan-3-ols to trans-flavan-3ols did not occur under our in vitro assay conditions. The optimal $\mathrm{pH}$ (using citrate/phosphate and Tris- $\mathrm{HCl}$ buffers) and temperature for PsANR activity were determined to be 7.0 and $40^{\circ} \mathrm{C}$, respectively. In the optimized reaction condition, the kinetics properties of PsANR for the three substrates were further determined (Figure 5 and Table 3). The rates of the respective product formation (i.e., cis-flavan-3-ol) from substrates fit well to the Michaelis-Menten kinetics model with minor variations in affinity and turnover number. PsANR showed comparable $k_{\text {cat }}$ values for all three substrates ranging from 0.5 to $1.2 \times 10^{-3} \mathrm{sec}^{-1}$. However, the $K_{m}$ values for pelagonidin and cyanidin as substrates were approximately 5-fold lower than for delphinidin, making the overall kinetic efficiency of PsANR for delphinidin 2-7 fold lower than for pelagonidin and cyanidin. Interestingly, it was recently reported that ANRs from Vitis vinifera (grape) and Camellia sinensis (tea) have an intrinsic epimerase activity, producing trans-flavan-3ols in vitro as well as cis-flavan-3-ols [32,33]. Of interest to this study is the possibility that ANR could contribute to the formation of trans-flavan-3-ols, along with its known ability to form cis-flavan-3-ols. However, we observed no evidence for PsANR epimerase activity for the conversion of cis-flavan-3-ols to trans-flavan-3-ols, as trans-flavan-3-ol products were not observed using cis-flavan-3-ols (EC and EGC) as substrates in the PsANR recombinant enzyme assays (Additional file 3: Figure S2).

\section{Transcriptome of $P$. sativum seed coat}

Although ANR activity could be evaluated using commercial substrates, LAR substrates (leucoanthocyanidins; Figure 1) are not stable or commercially available. Due to the lack of substrate, LAR activity was examined using enzymatically synthesized substrates by the DFR recombinant enzyme. However, both PsDFR and PsLAR clones were not present in the publicly available EST database. During the progress of this work, two Transcript Shotgun Assembly (TSA) data from garden and field pea were released to the NCBI using next-generation sequencings (NGS, Roche/454 sequencing platform) [34,35]. In these data sets, a full length PSDFR could be identified, but a PsLAR clone was still missing since the seed coat was not included in these sequencing samples.

To improve the current pea TSA data and also to facilitate the present studies of PA metabolism, pea seed coats were physically isolated and pooled from ripening fruits between 10 and 25 DAA. A small scale NGS (a quarter plate) was performed using the Roche/454 sequencing method. Accordingly, a total of 40,903 reads with an average of 392-bp read length were generated, and 
Figure 4 Temporal profiles of PsANR, PsDFR and PsLAR transcript abundance, PA content and mean degree of polymerization in pea seed coats of 'Courier'. A) PA content (black circles) and mean degree of polymerization (mDP: white circles) in developing 'Courier' seed coats from 12 to 30 DAA; data are means \pm SE $(n=3)$. Relative transcript abundance of 'Courier' B) PSANR C) PSDFR and D) PSLAR from 6 to 20 DAA using qRT-PCR. Transcript abundance values of PSANR and PSDFR were normalized to the 20 DAA, and PSLAR to the 12 DAA samples. Actin was used as the reference gene in all experiments. Data are means $\pm \mathrm{SE}$ (PSLAR and PSANR, $\mathrm{n}=4$; PSDFR $\mathrm{n}=3$ ).

these individual reads were assembled through MIRA algorithm to yield 16,272 unigenes (5,766 contigs and 10,506 singletons) [36]. These unigenes were annotated by BLASTx against TAIR and UniProt protein sets through the FIESTA bioinformatics pipeline (Plant Biotechnology Institute, Canada). With an E-value of $10^{-2}$ cut-off, the unigenes showed 9,702 and 9,420 hits against TAIR and UniProt protein sets.

Annotated unigenes were ranked by their abundance, according to the number of reads constituting the contigs (Additional file 4: Table S2). The transcripts among the top 20 highly expressed genes included 1-aminocyclopropane1-carboxylate oxidase (ethylene biosynthesis; ranked 2nd), indole-3-acetic acid amido synthetase (auxin sequestering, ranked 3rd), methionine synthase (ethylene biosynthetic precursor; rank 4 th), and gibberellin $2 \beta$-dioxygenase (PsGA2ox1, gibberellin deactivation gene; ranked 16th). These results are consistent with gene expression changes observed in other studies (increase in PsGA2ox1 in the pea seed coat during a similar stage of development [37] and other hormonal regulation of seed development processes [38]). It should be noted that two unigenes annotated as ANR and F3'5' hydroxylase were ranked as the $5^{\text {th }}$ and $6^{\text {th }}$ most abundant contigs in the database, indicating that PA biosynthesis is a major metabolic route in pea seed coat.

Next, we assessed the coverage of PA metabolic genes represented in our seed coat-specific TSA data set. The protein sequences of the characterized enzymes involved in PA biosynthesis were curated from Arabidopsis, Medicago sativa (alfalfa), M. truncatula, and petunia (Petunia spp.), and were used as BLASTx queries. The identified contigs and singletons with high E-value hits were manually inspected to determine the numbers of reads for each gene. This quantitative analysis revealed that all 12 genes for PA biosynthesis are present in the pea TSA data set, but their read numbers varied significantly (from 2 to 222 out of $\sim 40,000$ total reads; Figure 1, numbers in parenthesis). In agreement with the PA chemical phenotype of 'Courier' (mostly 3',4',5'-hydroxy flavan-3-ols), F3'5' $H$ showed an abundant read number (186 reads) of transcripts while $F 3^{\prime} H$ had only two reads. As these two enzymes compete for the common substrate naringenin, this relative transcript abundance explains the delphinidin- 

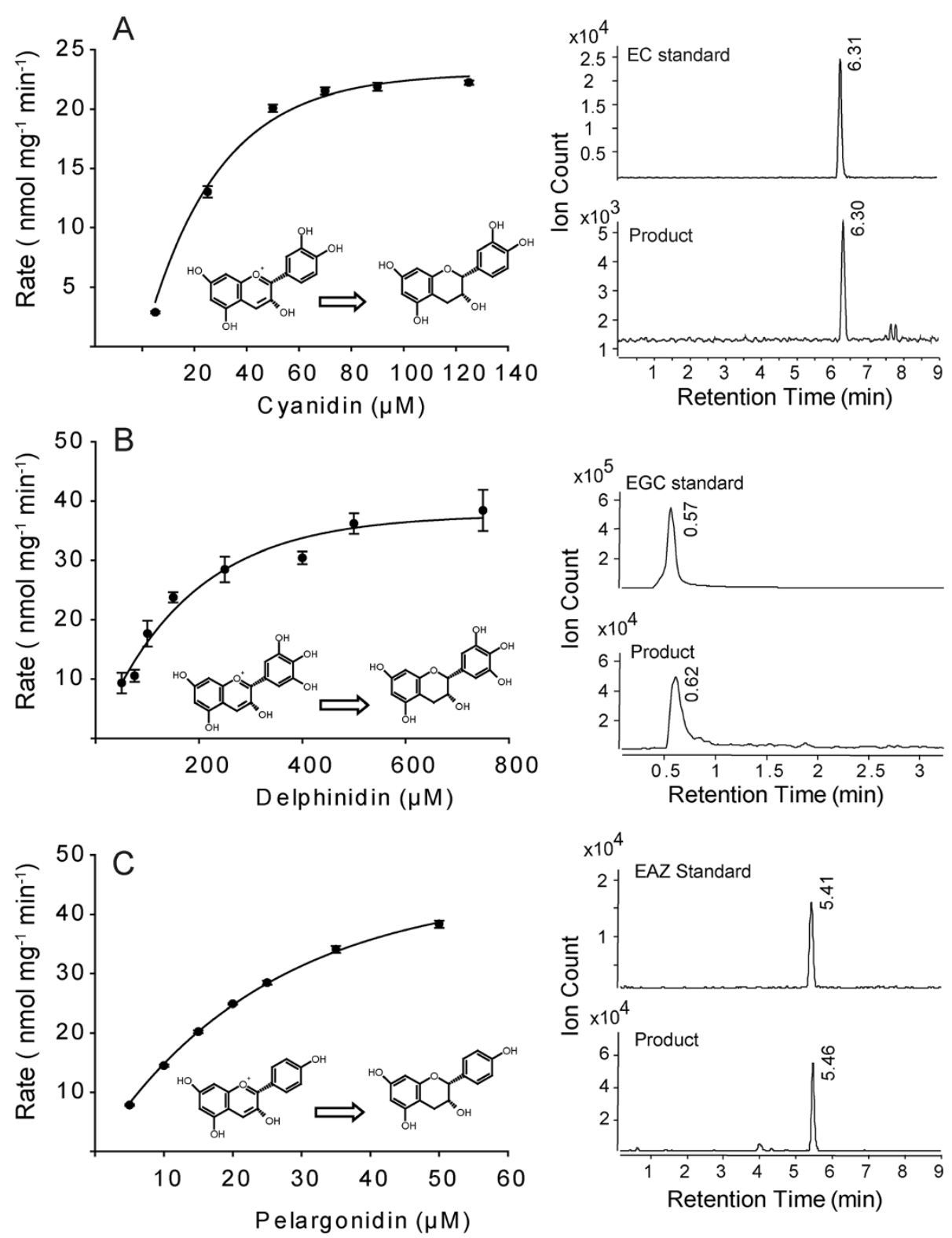

Figure 5 In vitro characterization of PsANR recombinant enzyme. A-C: PSANR reaction kinetics were explored using cyanidin (A), delphinidin (B) and pelargonidin (C). Left: Michaelis-Menten kinetics plots. Each data point represents means \pm SE $(n=3)$. Right: LC-MS identification in reference to authentic standards $[(-)$-epicatechin $(\mathrm{m} / \mathrm{z}=291)$, (-)-epigallocatechin $(\mathrm{m} / \mathrm{z}=307)$, and $(-)$-epiafzelechin $(\mathrm{m} / \mathrm{z}=275)]$.

Table 3 PsANR reaction kinetics using cyanidin, pelargonidin or delphinidin as a substrate

\begin{tabular}{|c|c|c|c|c|}
\hline Substrate & $\mathrm{K}_{\mathrm{m}}(\mu \mathrm{M})$ & $\mathrm{V}_{\max }\left(\mathrm{nmol} \mathrm{mg}^{-1} \min ^{-1}\right)$ & $k_{\text {cat }}\left(\sec ^{-1}\right)$ & $k_{\text {cat }} / \mathrm{K}_{\mathrm{m}}\left(\mathrm{M}^{-1} \mathrm{sec}^{-1}\right)$ \\
\hline Pelargonidin & $39.0 \pm 0.1^{a}$ & $72.5 \pm 2.2^{a}$ & $1.2 \times 10^{-3}$ & 30.7 \\
\hline Cyanidin & $37.0 \pm 0.2$ & $30.2 \pm 5.0$ & $0.5 \times 10^{-3}$ & 13.5 \\
\hline Delphinidin & $183.6 \pm 0.2$ & $47.3 \pm 6.4$ & $0.8 \times 10^{-3}$ & 4.3 \\
\hline
\end{tabular}

${ }^{\mathrm{a}}$ Data are means $\pm \operatorname{SE}(\mathrm{n}=3)$. 
derived PA subunits in 'Courier'. In this TSA data set, DFR and $A N R$ were represented by 71 and 222 reads, respectively, and they were present as full-length genes. However, $L A R$ had only 13 reads and was present as a partial clone (Figure 1).

\section{Cloning and characterization $P$. sativum LAR}

The deduced protein sequences from the full-length PsDFR (1,029-bp ORF) is approximately $38.4 \mathrm{kDa}$, and it shows $89 \%$ and $70 \%$ amino acid identity to $M$. truncatula and Arabidopsis DFR, respectively. Contigs representing PSLAR lacked a portion of the 5'-sequence, and hence the full-length PSLAR (1,056-bp ORF) was recovered by 5'RACE. The encoded PsLAR protein sequence, calculated to be approximately $38.8 \mathrm{kDa}$, is $85 \%$ and $67 \%$ identical to M. truncatula LAR and Desmodium uncinatum LAR, respectively. The LAR characteristic amino acid motifs RFLP, ICCN, and THD were conserved in the PsLAR protein sequence (Additional file 5: Figure S3) [39].
To examine their catalytic activities, PsLAR and PSDFR were expressed as recombinant proteins with $\mathrm{N}$-terminal six-histidine tags and purified using the same method as for PsANR (Additional file 6: Figure S4). Purified PsDFR recombinant enzyme was used to provide PsLAR substrate in vitro. In these coupled assays, purified PsDFR and PsLAR were mixed at a 2:1 molar ratio, and the DFR substrate, dihydroquercetin (DHQ) or dihydromyricetin (DHM), was added to the reaction assays in optimized reaction conditions $\left(40^{\circ} \mathrm{C}\right.$ and a slightly acidic $\mathrm{pH}$ of 6$)$. The formation of predicted trans-flavan-3-ol (CT or GC) was then analyzed by LC-MS in comparison to the authentic standards (Figure 6). Only co-incubation of PsDFR and PsLAR could synthesize compounds displaying $[\mathrm{M}+\mathrm{H}]^{+}$ion for CT $(m / z=291)$ or GC $(m / z=307)$ (Figure 6C and E).

Overall, the coupled assays showed very efficient conversions of the substrates, DHQ and DHM. When the coupled assays were performed at $65 \mu \mathrm{M}$ substrate, $36 \%$ conversion of DHQ to CT and $12 \%$ conversion of DHM to GC were observed. Despite the inaccuracy to calculate
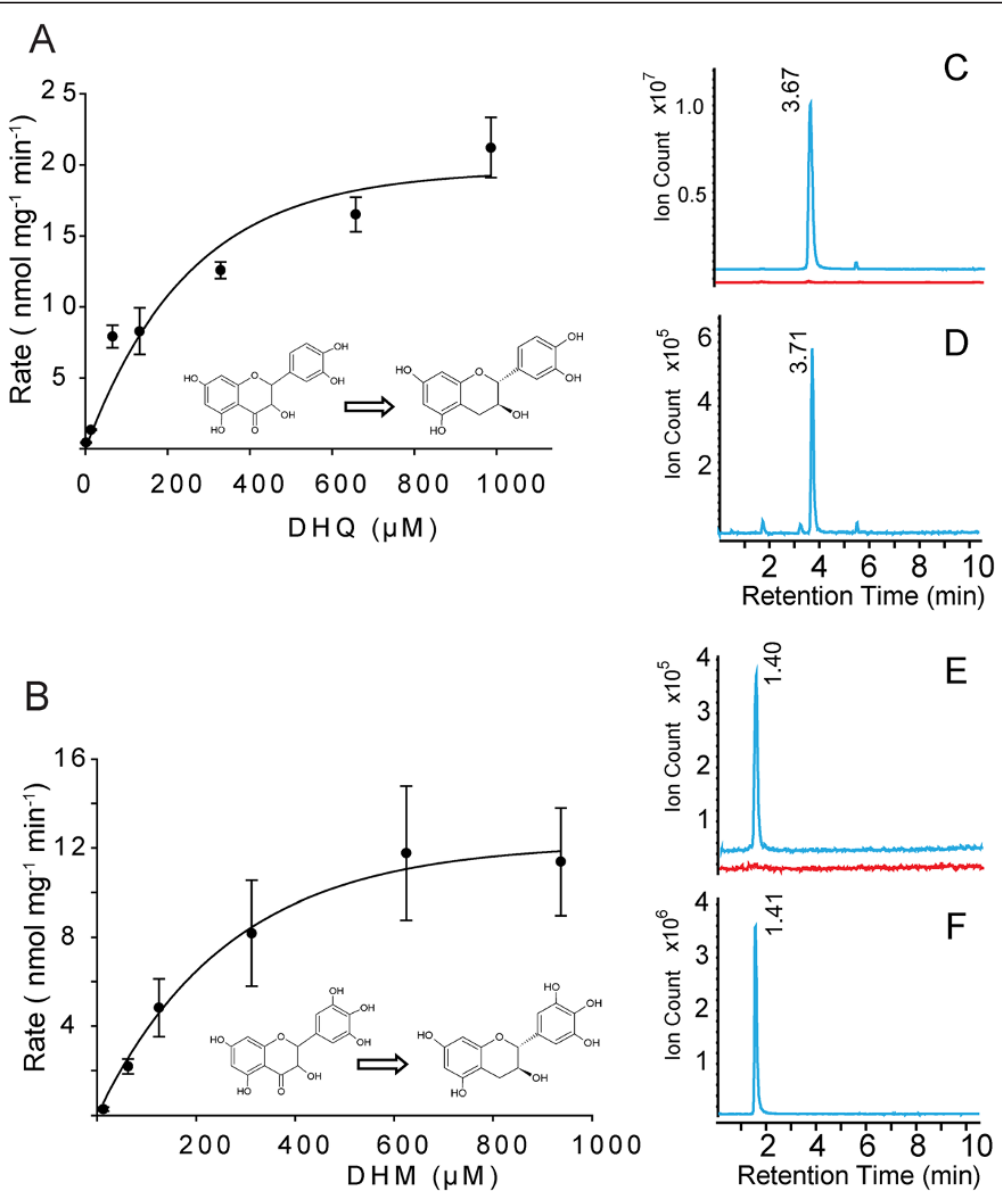

Figure 6 In vitro PsDFR and PsLAR coupled assays. Product synthesis rates from the coupled assays were measured using DFR substrates, dihydroquercetin (A) and dihydromyricetin (B). Left: pseudo-kinetics plots were inferred from the coupled assays. Each data point represents means \pm SE $(n=3)$. C-F: LC-MS $[M+H]^{+}$extracted ion chromatographs ( $C$ and $\mathbf{D} \mathrm{m} / \mathrm{z}=291 ; \mathbf{E}$ and $\left.\mathbf{F}, \mathrm{m} / \mathrm{z}=307\right)$ of authentic ( + )-catechin (D) and $(+)$-gallocatechin $\mathbf{( F )}$ along with in vitro assay products (C and E) from PsDFR only (red line) or PsDFR + PsLAR coupled assays (blue line). 
kinetic properties from the coupled assays, PsLAR kinetic values were inferred by plotting product formation rate in relation to varying substrate (DHM and DHQ) concentrations. In these pseudo-kinetic analyses, the product synthesis rates $\left(V_{\max }\right)$ of the coupled assays were 2 to 3 -fold lower than those from ANR but still comparable (Figure 6 and Table 3).

As observed for PsANR activity, the substrate with a lower degree of B-ring hydroxylation (DHQ) was converted more efficiently, even though DHM is the expected native substrate in 'Courier' seed coats. For DFR, LAR and ANR, the degree of B-ring hydroxylation of the available substrate is determined by the upstream activity of flavonoid 3'-hydroxylase (F3'H) and flavonoid 3'5'-hydroxylase (F3'5'H) (Figure 1). Preliminary gene expression data from our lab (unpublished) indicates that $P S F 3^{\prime} H$ is highly expressed in 'LAN3017' versus F3'5' $\mathrm{H}$ in 'Courier' and 'Solido', which matches the observed PA profiles (Table 1). Thus, substrate availability is controlled independently from the substrate preference of these enzymes. The confirmation of an active PsLAR protein when coupled to PsDFR in vitro, therefore, supports the abundance of 2,3-trans-flavan-3-ols found in the pea seed coats.

\section{Developmental regulation of PsANR and PsLAR in 'Courier' seed coats}

With the demonstration of enzymatically competent PsANR and PsLAR, we assumed that coordinated expression of PSANR and PSLAR determines the PA content and composition in pea seed coats of 'Courier'. To understand developmental regulation of these two key genes, temporal expression of PSANR and PSLAR from 6 to 20 DAA in 'Courier' seed coat was determined by quantitative realtime PCR (qRT-PCR; Figure $4 \mathrm{~B}$ and C). The transcript abundance of both PSANR and PSLAR was high during the earlier stages of pea seed coat development. Both genes displayed a decline in expression as the seed coat matured, but PsLAR transcripts decreased significantly faster than PSANR transcript. Seed coat PSDFR transcript levels (codes for the enzyme responsible for the production of substrate used by LAR and ANS) were stable from 6 to 20 DAA, except for a 2-fold increase at 10 DAA (Figure 4D). Maximal PA accumulation in 'Courier' seed coat did not immediately follow transcriptional induction of PSANR and PSLAR, but it reached its highest level at 20 DAA (Figure 4A). PA mDP increased to five by $15 \mathrm{DAA}$, and it remained at this level to $20 \mathrm{DAA}$ (Figure 4A).

\section{Heterologous expression of PsLAR in Arabidopsis}

Arabidopsis lacks $L A R$ and does not synthesize transflavan-3-ols. In Arabidopsis, all leucoanthocyanidins are channelled to cis-flavan-3-ols by ANS and ANR. In order to examine if the expression of PSLAR in Arabidopsis seed coat can re-direct the metabolic flux to trans-flavan-3-ols,
PSLAR was expressed with a FLAG-epitope tag by a $~ 1.3-$ $\mathrm{Kb}$ fragment of Arabidopsis ANR promoter [27]. This construct $\left(P_{A N R}-P S L A R\right)$ was transformed to wild-type Arabidopsis as well as ANR knock-out (anr) Arabidopsis mutants identified from a T-DNA knock-out database. We hypothesized that the wild-type Arabidopsis expressing PSLAR would synthesize PAs comprised of a mixture of cis- and trans-flavan-3-ols while the Arabidopsis anr mutant expressing PSLAR would produce PAs exclusively composed of trans-flavan-3-ols.

Using transgenic plants, T-DNA insertions were confirmed by PCR-screening of genomic DNA in the anr mutant (data not shown). Subsequently, presence of PSLAR transcript and its recombinant enzyme were confirmed by RT-PCR and immunoblot analysis using anti-FLAG antibodies (Additional file 7: Figure S5A/B). Furthermore, activity of the PsLAR was confirmed using crude protein extracted from siliques with and without supplementary recombinant PsDFR (Additional file 7: Figure S5C). Therefore, the transgenic Arabidopsis produced functional LAR. Subsequently, the Arabidopsis transgenic lines were examined for alteration of PA subunit chemical phenotypes. The anr mutant expressing PSLAR was used to test for restoration of seed coat color and to detect the presence of DMACA reactive products; the seeds from wild-type Arabidopsis expressing PSLAR were used to profile monomer units of PA after phloroglucinol derivatization. Despite the clear evidence of successful transformation and presence of functional PsLAR, no complementation of the seed coat color or presence of DMACA reactive products were observed in the anr mutant background, nor was the presence of trans-flavan-3-ol and its derivatives observed in wild-type Arabidopsis (Additional file 8: Figure S6).

\section{Discussion}

Proanthocyanidin biosynthesis in pea (Pisum sativum) seeds To investigate the PA diversity of pea seeds, the PA profiles of four pea cultivars were analyzed, and significant quantitative and qualitative variations in PA chemistry were observed. Of the cultivars examined, 'Canstar' lacked detectable PAs, while 'Courier', 'Solido', and 'LAN3017' had different quantities and/or types of PAs (Table 1; Figure 3). All three PA-containing cultivars contained PA levels comparable to that found in blueberries, cranberries, sorghum (high tannin whole grain extrudate) and hazelnuts [40]. 'Courier' and 'Solido' PAs are composed primarily of prodelphinidin subunits (tri-hydroxylated B-ring; Figure 1; Table 1), similar to the pea cultivars 'CDC Acer' and 'CDC Rocket' [41], and those found in tea [42]. In contrast, 'LAN3017', was composed of procyanidin-type subunits (di-hydroxylated B-ring; Figure 1; Table 1). These differences in PA subunit composition may impact the nutritional quality as tri-hydroxylated flavan-3-ols (e.g. GC and EGC) have a higher antioxidant potential than di-hydroxylated 
forms (e.g. CT and EC) [43]. Additionally, the mean degree of polymerization of 'LAN3017' PAs are 2-3 fold higher than 'Courier' or 'Solido' PAs. The mechanism controlling PA polymerization remains unknown, but it is particularly relevant as bioavailability after consumption by animals and humans is inversely related to polymer length [4]. In this regard, pea offers a valuable system to investigate the molecular basis for subtle biochemical differences in PA biosynthesis, and the pea cultivars with different PA profiles can be integrated into animal and human nutritional studies.

Although delphinidin-derived epi-gallocatechin is the most abundant cis-flavan-3-ol subunit in the PAs of 'Courier' (Table 1), the PA precurors substrates, pelargonidin and cyanidin, were utilized more efficiently than delphinidin by recombinant PsANR in our in vitro assays (Table 3). In comparison, PAs of tea tree (Camellia sinensis) also contain a high proportion of delphinidin-derived flavan-3ol subunits, and its ANR displayed a higher preference for delphinidin than the other two substrates, displaying consistent in vivo and in vitro substrate preference [33]. The discrepancy of substrate preference found in pea 'Courer' is enigmatic, but obviously substrate availability for PsANR dictates PA monomer types in pea. It is well established that relative expression of $F 3^{\prime} \mathrm{H}$ and $F 3^{\prime} 5^{\prime} \mathrm{H}$ determines the PsANR substrate availability. We speculate that a dominant expression of $F 3^{\prime} 5^{\prime} H$ may have recently occurred in 'Courier', but downstream PsANR has not fully adapted to delphinidin as a preferred substrate in this cultivar.

\section{Contribution of $L A R$ to proanthocyanidin biosynthesis in pea and other plants}

With the discoveries of ANR (or BANYULS) from Arabidopsis [12] and LAR from D. uncinatum [13], it has been well accepted that cis-flavan-3-ols are synthesized by the consecutive reactions of ANS and ANR, and transflavan-3-ols are synthesized by LAR, from the common substrates flavan-3,4-diols (leucoanthocyanidins) (Figure 1). For LAR activity, biochemical data using purified recombinant LAR enzyme from this work and others (D. uncinatum [13], grape [39], and tea [33]) have shown that LAR efficiently catalyzes the synthesis of trans-flavan-3-ols from leucoanthocyanidins (e.g., leucocyanidin). In the DFR/LAR coupled assays shown here, the synthesis rates of the LAR products, catechin and gallocatechin, from their respective substrates (i.e., DFR substrate) were slightly lower than, but still comparable to, those of cis-flavan-3-ols by ANR. These data are consistent with the pea PA monomer profile composed of comparable amounts of trans- and cis-3-flavan-3ols. Although $L A R$ has been isolated from several plants, LAR kinetic data is scarce, due to the instability and inaccessibility of its substrates. The only $K_{m}$ values reported are $5-26 \mu \mathrm{M}$ for three types of leucoanthocyanidins from native D. uncinatum LAR [13]. In DFR/LAR coupled assays, the amount of LAR substrates (i.e. intermediates in coupled reaction) is expected to be very low. Thus, the efficient synthesis of PsLAR products observed in the coupled assays implies rapid consumption of low abundant intermediates by PsLAR and may reflect high affinity of the substrates to PsLAR.

The biochemical data for PsLAR strongly support its role in production of trans-flavan-3-ols in pea. However, data from $L A R$ overexpression studies in heterologous plants suggest that production of trans-flavan-3-ols through LAR in planta may require more than the presence of enzymatically competent LAR protein. Previously, expression of $D$. uncinatum and M. truncatula $L A R$ by constitutive viral promoters in two LAR-lacking (thus, trans-flavan-3-ol-free) plants, white clover (Trifolium repens) and tobacco (Nicotiana tabaccum), did not lead to the production of trans-flavan-3-ols $[13,26]$. In the present study, instead of using a constitutive promoter, the Arabidopsis ANR promoter (known to drive strong gene expression in the seed coat) was used to express PSLAR in the Arabidopsis seed coat [27]. We hypothesized that the $A N R$ promoter will express PsLAR at appropriate developmental stages in seed coat cells, where substrates for PsLAR are abundant. Accordingly, PsLAR transcript, protein, and catalytic activity were clearly detected from the transgenic Arabidopsis siliques (Additional file 7: Figure S5); nonetheless, no restoration of seed coat PA phenotype was observed in anr mutant Arabidopsis, and no catechin PA extension or terminal subunits could be detected after phloroglucinolysis analysis from $L A R$ overexpressing wild-type Arabidopsis (Additional file 8: Figure S6). This result suggests that simply placing active PsLAR enzyme in the seed coat could not sufficiently redirect the metabolic flux toward trans-flavan-3-ols. With our data in mind, it is noteworthy that a protein complex channelling dihydromyricetin (DFR substrate) to gallocatechin (LAR product) was purified from forage legume, Onobrychis viciifolia [44]. Therefore, although speculative, PsLAR may need to form an enzyme complex with PsDFR in vivo to fully draw a metabolic flux towards trans-flavan-3-ol synthesis. Further studies are required to test this model in pea or transgenic Arabidopsis.

Both PSLAR and PSANR transcript abundance was high earlier in pea seed coat development (Figure $4 B$ and $C$ ). PSLAR showed the highest expression at 6 DAA and its transcripts were rapidly reduced to a basal level by 10 DAA. PSANR displayed a wider range of expression with substantial transcript levels until 15 DAA. By 12 DAA, PA accumulation in the seed coat was approximately halfmaximal, reaching maximal levels at 20 DAA (Figure 4A). Consistent with this result, the 454-sequencing read number of PSLAR from the 10-25 DAA seed coat samples was 17-fold lower than that of PSANR (Figure 1). Curiously, 
the molar percent of GC extension units (LAR product) in 'Courier' seed coat PAs steadily increased from 12 DAA to 30 DAA, but PSLAR transcript abundance was minimal by 12 DAA (Table 2; Figure 4C). This apparent inconsistency between PSLAR expression and GC incorporation in pea seed coat PAs suggests that a pool of flavan3 -ols is made earlier in seed coat development, and this pool supplies substrates for PA polymerization throughout the remainder of tissue development. Alternatively, PsLAR protein may have an unusually long half-life. An understanding of the mechanism of PA polymerization is required to better address this discrepancy. Intriguingly, it was reported that grape ANR can synthesize not only EC (cis-flavan-3-ol) but also CT (trans-flavan-3-ol) in 50:50 molar ratio by its intrinsic epimerase activity [32], and such epimerase activity was also recently observed in tea ANR [33]. However, epimerase activity for the conversion of cisflavan-3-ols to trans-flavan-3-ols could not be found from PsANR in our study, suggesting the trans-flavan-3-ols in pea were not derived from PsANR epimerase activity.

Taking all data together from this work and others, LARs from different plants have displayed competent biochemical activity to transform leucoanthocyanidins to trans-flavan-3-ols in vitro; however, the lack of accumulation of trans-flavan-3-ols and their derivatives accompanying expression of $L A R$ in heterologous $L A R$-free plants still raises questions. It appears that the analysis of a $L A R$ knock-out in trans-flavan-3-ol abundant plants (e. g., pea, tea, and grape) is necessary to definitely establish in vivo function of $L A R$.

\section{Conclusions}

In this report, our comprehensive chemical analyses of PAs from pea seed coat showed that pea PAs are composed of both cis- and trans-flavan-3-ols and that substantial quantitative and qualitative variations of PA subunits (e.g., degree of hydroxylation and polymerization) are present among different pea cultivars. The transcriptomics analysis of the PA-rich 'Courier' seed coat identified key biosynthetic genes for both cis- and trans-flavan3-ol synthesis in agreement with the PA profile in pea. The catalytic identities of the two key genes for PA synthesis (PSANR and PSLAR) were further confirmed by biochemical assays. Despite potent in vitro activity of PsLAR, expression of PSLAR in Arabidopsis seed coat (both in wild-type and $A N R$ knock-out backgrounds) was unable to redirect the metabolic flux towards trans-flavan-3-ol synthesis, implying a possible in planta metabolite channelling. We expect that the improved understanding of PA chemical variations and associated biosynthetic mechanisms will help us develop pea cultivars with desirable PA types and quantity.

\section{Methods}

\section{Plant material and growth conditions}

Mature air-dried seeds of the pea (Pisum sativum L.) cultivars, 'Canstar', 'Courier', 'Solido' and 'LAN3017' (grown in Lethbridge, Alberta, Canada in 2007 or in Barrhead or Namao, Alberta, Canada in 2008) were used for PA extraction or growth chamber studies. For growth chamber studies, seeds were planted at an approximate depth of $2.5 \mathrm{~cm}$ in 3-L plastic pots (3 seeds per pot) in Sunshine no. 4 potting mix (Sun Gro Horticulture, Vancouver, Canada) and sand at 4:1. Plants were grown in a climate-controlled growth chamber with a $16 \mathrm{~h}$-light/ $8 \mathrm{~h}$-dark photoperiod $\left(19^{\circ} / 17^{\circ} \mathrm{C}\right)$ with an average photon flux density of 383.5 $\mu \mathrm{E} / \mathrm{m}^{2} / \mathrm{s}$ (measured with a LI-188 photometer, Li-Cor Biosciences, Lincoln, Nebraska). Flowers were tagged at anthesis, and seeds were harvested at selected stages as identified by days after anthesis (DAA). Seeds were harvested directly onto ice at $6,8,10,12,15,20,25$, and 30 , DAA and dissected immediately into seed coats and embryos and then stored at $-80^{\circ} \mathrm{C}$.

\section{RNA isolation and CDNA preparation}

For cloning and qRT-PCR assays, total RNA was isolated from 'Courier' pea seed coat tissue using a Qiagen RNeasy Plant Mini kit. Polyvinylpyrrolidone (PVP-40) was added to the extraction buffer at a final concentration of $2 \%$ $(\mathrm{w} / \mathrm{v})$ to reduce precipitation of RNA by phenolic compounds contained in the seed coats [45]. First-strand cDNA was synthesized using Superscript II reverse transcriptase and an Oligo-d $\mathrm{dT}_{12-18}$ primer (Invitrogen). Synthesis was conducted according to the manufacturer's protocol.

\section{Cloning of the pea flavonoid genes $A N R, D F R$ and $L A R$}

PSANR was cloned from cDNA prepared from 20 DAA 'Courier' seed coat tissue using degenerate primers (Additional file 9: Table S3) based on conserved amino acid regions of ANR in Medicago truncatula (AAN77735.1), Malus $x$ domestica (AAZ79363.1), Fragaria ananassa (ABG76843.1), Arabidopsis thaliana (AAF23859.1) and Vitis vinifera (AAZ82409.1). PCR reactions $(50 \mu \mathrm{L})$ were run for a total of thirty-five cycles using 1 unit of Phusion polymerase (New England Biolabs), GC buffer (New England Biolabs), $0.2 \mathrm{mM}$ dNTPs and 2 pmol forward and reverse primers, and $250 \mathrm{ng}$ of 20 DAA Courier seed coat cDNA. Amplified fragments were cloned into pBlueScript II SK (-) (Stratagene) and sequenced. The fulllength sequence of PSANR was recovered by rapid amplification of cDNA ends (RACE) using a SMART RACE cDNA Amplification kit (Clontech). RACE fragments were ligated into a pGEM-T Easy vector (Promega) and sequenced. Fulllength PSANR was cloned into the Gateway donor vector pDONR221 (Invitrogen). The complete ORF of PsDFR was retrieved from the Courier 454-pyrosequencing data. The PSLAR sequence in the Courier 454-sequencing data lacked 
the 5'-end; therefore, 5'-RACE was performed to obtain the complete transcript sequence. The 5'-RACE fragment was ligated into a pGEM-T Easy vector (Promega) and sequenced. Full-length PSDFR and PSLAR were cloned into pDONR221 (Invitrogen) vector according to the manufacturer's protocol. See supplementary materials for primer sequences.

\section{Recombinant expression and purification of pea ANR, DFR and LAR}

Using the Gateway vector system, PSANR, PsDFR and PSLAR were cloned into pDEST17 (Invitrogen), containing an N-terminal 6x histidine tag for purification, and then transformed into E. coli BL21-AI (Invitrogen). The bacteria were grown in LB ampicillin $\left(100 \mu \mathrm{g} \mathrm{mL} \mathrm{m}^{-1}\right)$ media in a shaker at $37^{\circ} \mathrm{C}$ to an $\mathrm{OD}_{600}$ of 0.4-0.6 at which point the cultures were transferred to a refrigerator shaker for an additional $20-30$ minutes at $12^{\circ} \mathrm{C}$ (ANR) or $15^{\circ} \mathrm{C}$ (DFR and LAR). Expression was then induced by adding L-arabinose (Sigma) to a final concentration of $0.2 \%(\mathrm{w} / \mathrm{v})$. Following overnight incubation at $12^{\circ} \mathrm{C}$ or $15^{\circ} \mathrm{C}$, the bacteria were pelleted by centrifugation at $4^{\circ} \mathrm{C}$ at $8000 \times$ g for $20 \mathrm{~min}$. Pellets were resuspended in $1 \%$ original culture volume in lysis/wash buffer 1 (100 mM Tris- $\mathrm{HCl}$ pH 8, $10 \mathrm{mM}$ imidazole, $10 \%$ glycerol, $0.1 \%$ Triton X-100, $10 \mathrm{mM} \beta$ mercaptoethanol). Cells were lysed by sonication on ice using a Microson Ultrasonic Cell Disrupter XL (Misonix, Farmingdale, NY) at 6 Watts for 10 seconds, repeated 8-10 times. The lysate was centrifuged at $12,000 \times \mathrm{g}$ at $4^{\circ} \mathrm{C}$ for $20 \mathrm{~min}$. For PsANR and PsDFR purification, the supernatant was applied to a $1 \mathrm{~mL}$ Bio-Scale Mini Profinity IMAC column (BioRad) using a BioLogic DuoFlow (Biorad) fast protein liquid chromatography (FPLC) machine. The column was washed at $1 \mathrm{~mL} \mathrm{~min}^{-1}$ with $6 \mathrm{~mL}$ lysis/wash buffer 1 followed by $6 \mathrm{~mL}$ wash buffer $2(100 \mathrm{mM}$ Tris- $\mathrm{HCl}$ pH 8, 20 mM imidazole, 10\% glycerol, 0.1\% Triton X-100, $10 \mathrm{mM} \beta$-mercaptoethanol, $1 \mathrm{M} \mathrm{KCl}$ ). Recombinant protein was eluted at $1 \mathrm{~mL} \mathrm{m^{-1 }}$ with $7.5 \mathrm{~mL}$ of elution buffer (100 mM Tris- $\mathrm{HCl}$ pH 8, 250 mM imidazole, 10\% glycerol, $300 \mathrm{mM} \mathrm{KCl}$ ). The eluent was concentrated using an Amicon Ultra-30 column (Millipore). Aliquots of concentrated protein were immediately frozen in liquid nitrogen and stored at $-80^{\circ} \mathrm{C}$. Stability issues were encountered with PsLAR, and the recombinant enzyme was purified using a different method. After sonication and centrifugation, the supernatant was mixed for $1 \mathrm{hr}$ on ice with nickel-NTA resins (Bio-rad) previously equilibrated with buffer (100 mM Tris- $\mathrm{HCl}$ (pH 8), 10\% glycerol, $0.1 \%$ Triton X-100, supplemented with a protease-inhibitor cocktail (Roche)) containing $20 \mathrm{mM}$ imidazole. The mixture was then loaded onto a Poly-Prep chromatography column (Bio-rad) and washed with $10 \mathrm{~mL}$ of buffer containing $20 \mathrm{mM}$ imidazole. The column was eluted with $2 \mathrm{~mL}$ of buffer containing increasing concentrations of imidazole (50 mM, 100 mM, 200 mM, and 500 mM). Purity was checked by SDS-PAGE.

\section{PsANR in vitro assays}

Substrates used were pelargonidin, cyanidin, and delphinidin (all from Extrasynthase, Genay, France). Authentic standards used were (-)-epiafzelechin (MicroSource, Gaylordsville, Connecticut) and (-)-epicatechin and (-)-epigallocatechin (both from Extrasynthase). To determine the linear range of PsANR activity, 5-100 $\mu \mathrm{g}$ of purified concentrated protein was assayed in a final reaction volume of $250 \mu \mathrm{L}$. The assays were run in $100 \mathrm{mM}$ Tris- $\mathrm{HCl}$ containing $20 \mathrm{mM} \mathrm{NADPH}$ and $100 \mu \mathrm{M}$ cyanidin. Coumarin was added to a final concentration of $25 \mu \mathrm{M}$ as an internal standard. The reactions were incubated at $30^{\circ} \mathrm{C}$ for $30 \mathrm{~min}$ and stopped by extracting twice with $500 \mu \mathrm{L}$ ethyl acetate, vortexing for $1 \mathrm{~min}$, and centrifuging for $1 \mathrm{~min}$. The ethyl acetate was evaporated under a $\mathrm{N}_{2}$ stream. The organic fraction was resuspended in $50 \mu \mathrm{L}$ of $50 \%$ methanol and analyzed by high performance liquid chromatography (HPLC; Waters 2795 Separations Module) using a Sunfire C18 $3.5 \mu \mathrm{m} 4.6 \times 150 \mathrm{~mm}$ column (Waters) and a photodiode array detector scanning between 100-400 nm. Peak area was quantified at $280 \mathrm{~nm}$ (EC, EAZ) and $270 \mathrm{~nm}$ (EGC). For the cyanidin and pelargonidin assays, the column was eluted using a linear gradient consisting of solvent A $\left(100 \% \mathrm{H}_{2} \mathrm{O}\right)$ and solvent $\mathrm{B}(100 \%$ acetonitrile) at a flow rate of $1.2 \mathrm{~mL} \mathrm{~min}^{-1}$ as follows: 08 min $20-50 \%$ B, 8.5-10 min 100\% B. The chromatography gradient for delphinidin assays was $0-17 \min 5-50 \% \mathrm{~B}$, 17.01-18.5 min 100\% B. Optimum temperature for PsANR activity was determined at temperatures between $25-60^{\circ} \mathrm{C}$. Incubation time linearity was determined at $40^{\circ} \mathrm{C}$ for 15 , 30, 60, 90, 120 and $240 \mathrm{~min}$. Three buffers were used to test a pH range from 4-8.5 (50 mM citrate/phosphate: $\mathrm{pH} 4,5$, 6, 7; 50 mM MES (2-(N-morpholino) ethanesulfonic acid): $\mathrm{pH}$ 5, 6, 6.5, 7; 100 mM Tris-HCl: $\mathrm{pH}$ 7, 7.5, 8, 8.5). Kinetics assays were carried out in triplicate in a total reaction volume of $250 \mu \mathrm{L}$ containing $100 \mathrm{mM}$ Tris- $\mathrm{HCl}$ $\mathrm{pH} 7$, using $40 \mu \mathrm{g}$ ANR, $20 \mathrm{mM} \mathrm{NADPH}$, at $42^{\circ} \mathrm{C}$ for 20 minutes.

\section{Recombinant PsDFR-PsLAR coupled enzyme assays}

Substrates used were dihydroquercetin (DHQ; Extrasynthase) and dihydromyricetin (DHM; Chromadex, USA). Authentic standards used were (+)-catechin and (-)-gallocatechin (both from Sigma). In vitro coupled enzyme activity assays were carried out using $100 \mathrm{mM}$ Tris- $\mathrm{HCl}, 50 \mu \mathrm{g}$ PsDFR, $25 \mu \mathrm{g}$ PsLAR, $100 \mu \mathrm{M}$ DHM, $2 \mathrm{mM}$ NADPH at temperatures from $22^{\circ} \mathrm{C}$ to $68^{\circ} \mathrm{C}$ for 30 minutes to determine the optimum temperature for the coupled reaction. The optimum $\mathrm{pH}$ of the coupled assay was determined using $50 \mathrm{mM}$ MES ( $\mathrm{pH}$ 5, 6, 7), $100 \mathrm{mM}$ sodium phosphate buffer $(\mathrm{pH} \mathrm{5.5,6,7)}$ or 100 mM Tris-HCl (pH 7, 7.5, 8, 8.5). $100 \mu \mathrm{M}$ DHM, $50 \mu \mathrm{g}$ PsDFR and $25 \mu \mathrm{g}$ PsLAR were added to each $250 \mu \mathrm{L}$ reaction. 
NADPH was added to a final concentration of $2 \mathrm{mM}$. HPLC analysis of ANR assay products were performed as described above. Acetosyringone was used as an internal standard.

\section{Arabidopsis PsLAR transgenic plants}

pKGWFS7 [46], contained a seed coat specific expression cassette ( $\mathrm{P}_{\mathrm{ANR}}:$ FLAG::PSLAR::T35S) generated by PCRstitching (see Additional file 9: Table S3 for primers), consisting of a 1367 bp portion of the native A. thaliana $B A N$ promoter, shown to be sufficient to drive seed coat specific expression of a GUS reporter [27]. Briefly, full-length PSLAR was cloned from 'Courier' 10 DAA cDNA using primers 15 and 16, the $\mathrm{P}_{\mathrm{ANR}}$ fragment was cloned from Arabidopsis Columbia-0 genomic DNA using primers 20 and 21, and T35S was cloned from pKWG2D [46] using primers 18 and 19, which also introduced a 5'-overlap region with 3'-PSLAR. PSLAR and T35S were stitched together using primers 17 and 19, which also added a 5'FLAG tag to PSLAR. A 3'overlap region with 5'-FLAG:: PSLAR was added to the $\mathrm{P}_{\mathrm{ANR}}$ fragment using primers 20 and 22. The $\mathrm{P}_{\mathrm{ANR}}:$ FLAG and FLAG::PSLAR::T35S constructs were stitched together using primers 19 and 23. PCR reactions $(50 \mu \mathrm{L})$ were run for a total of thirty cycles using 1 unit of Phusion polymerase (New England Biolabs), HF buffer (New England Biolabs), 0.2 mM dNTPs and 0.40.5 pmol forward and reverse primers. Touchdown PCR was used for the stitching reactions with an initial five cycles at an annealing temperature (Tm) dependent on the overlap regions involved. The $\mathrm{Tm}$ of the subsequent twenty-five cycles was dependent on the PCR primer Tm. Additionally, when stitching, the templates were added in equal molar ratios and PCR primers were added after the initial five cycles.

The vector was transformed into Agrobacterium tumefaciens GV3101. Arabidopsis Columbia-0 and an ANR T-DNA insertion line (SALK_040250C; ANR knock out line) were transformed by floral tip [47], T1 seeds were tested for kanamycin resistance and positive transformants were confirmed by genomic PCR using primers 16 and 20.

Activity of PsLAR in the transgenic lines was confirmed by in vitro assays using crude protein extracts. Immature siliques $(40 \mathrm{mg}$ ) were ground first in liquid nitrogen and then in $600 \mu \mathrm{L}$ of ice-cold extraction buffer (50 mM Tris$\mathrm{HCl}$ (pH 7), 1\% (w/v) PVP (polyvinylpyrrolidone), $0.2 \mathrm{mM}$ PMSF (phenylmethylsulfonyl fluoride), 10\% glycerol, $5 \mathrm{mM}$ sodium metabisulfite, and $1 \mathrm{mM}$ 2-mercaptoethanol). Following grinding, the mixture was centrifuged at $11,000 \times \mathrm{g}$ for $10 \mathrm{~min}$ at $4^{\circ} \mathrm{C}$. $150 \mu \mathrm{g}$ total soluble protein with or without $130 \mu \mathrm{g}$ of recombinant PsDFR was incubated in $50 \mathrm{mM}$ MES (pH 6) with $2 \mathrm{mM}$ NADPH and $100 \mu \mathrm{M}$ dihydroquercetin in a final volume of $500 \mu \mathrm{L}$. The reaction was incubated at $35^{\circ} \mathrm{C}$ for a total of $90 \mathrm{~min}$. Silique total soluble protein was added $30 \mathrm{~min}$ after the start of assay.
For LC-MS analysis, the reactions were stopped and extracted as described above.

\section{Liquid chromatography mass spectrometry (LC-MS/MS)}

PsANR reaction products were confirmed using an Agilent Technologies (Santa Clara, California) 6410 Triple Quad LC-MS/MS with a 1200 Series liquid chromatography system equipped with an electron spray ionization source and an Eclipse Plus C18 $1.8 \mu \mathrm{m} \mathrm{2.1 \times 50} \mathrm{mm} \mathrm{column} \mathrm{(Agilent).}$ Samples were extracted and prepared as described above. Products and standards were detected in positive ion mode using product ion scan. Mass to charge ratio $(\mathrm{m} / \mathrm{z})$ selected for fragmentation were: 291 for EC/CT, $307 \mathrm{EGC/GC}$ and 275 for EAZ. Fragmentor energies were: EC, 85 V; EGC, $50 \mathrm{~V}$; EAZ, $80 \mathrm{~V}$. Collision energies were: EC/CT, 12 and $20 \mathrm{eV}$; EGC/GC, 0 and $20 \mathrm{eV}$; EAZ, $20 \mathrm{eV}$. Liquid chromatography solvents were A) $1 \%(\mathrm{v} / \mathrm{v})$ aqueous acetic acid and B) acetonitrile. Gradients for the samples were: EAZ, 10-50\% B 0-8 minutes, 50-100\% B 8-10 minutes; EC/CT, 5\% B 0-0.5 minutes, 5-30\% B 0.5-8 minutes, 100\% B 8-11 minutes; EGC/GC $30-100 \%$ B 0-8 min. Flow rates for all samples were $0.4 \mathrm{~mL} \mathrm{~min}^{-1}$.

The products from in vitro assays using total soluble Arabidopsis silique protein were run on a longer gradient to ensure adequate separation between catechin and epicatechin; 0-40\% B 0-15 min, 40-100\% B 15-15.5 min, using $0.2 \%$ acetic acid in $5 \%$ acetonitrile (solvent A) and $0.2 \%$ acetic acid in $95 \%$ acetonitrile (solvent B).

\section{Real-time quantitative PCR}

Total RNA was extracted from 6-20 DAA pea seed coat tissue or immature Arabidopsis siliques as described above. Quantitative real-time PCR (qRT-PCR) was run on an Applied Biosystems StepOne machine using Power SYBR Green PCR mix (Applied Biosystems). A master mix containing SYBR Green and CDNA was prepared according to the manufacturer instructions and split evenly into plate wells containing $0.3 \mathrm{pmol}$ of gene forward and reverse primers such that each well contained 1-3 ng of cDNA (from mRNA) or 5-25 ng of cDNA (from total RNA). Relative transcript abundance was determined using the $\Delta \Delta \mathrm{C}_{\mathrm{T}}$ analysis method using Actin as the reference gene and three to four technical replicates [48].

\section{Extraction, purification and identification of proanthocyanidins}

To estimate seed coat total extractable PA concentration, seed coat tissue was lyophilized and ground to a fine powder using a Retsch ZM 200 mill (PA, USA) fitted with a $0.5 \mathrm{~mm}$ screen filter. For each sample, approximately $25 \mathrm{mg}$ of processed seed coat tissue was weighed into a $15 \mathrm{~mL}$ Falcon tube. The samples were extracted with $10 \mathrm{ml}$ of $80 \%$ methanol for $24 \mathrm{hr}$ with shaking. After vortexing the slurry and centrifuging for $5 \mathrm{~min}$ at $4000 \mathrm{rpm}$, the 
supernatants were used for PA analysis as previously described [31]. In brief, $2 \mathrm{~mL}$ of the butanol: $\mathrm{HCl}$ reagent and $66.75 \mu \mathrm{L}$ of iron reagent were added into a $15 \mathrm{~mL}$ glass culture tube. Then, $0.5 \mathrm{~mL}$ of clear sample extract was added to the tube and the mixture was vortexed. Two $350 \mu \mathrm{L}$ aliquots of the above solution were removed for use as sample blanks, and the remaining solution was placed into a $95^{\circ} \mathrm{C}$ water bath. After $40 \mathrm{~min}$ at $95^{\circ} \mathrm{C}$, the solution was allowed to cool at room temperature for $30 \mathrm{~min}$. The reaction products, sample blanks, and a PA standard curve dilution series were monitored for absorbance at $550 \mathrm{~nm}$ using a 96 well UV plate reader (Spectra Max 190, Molecular Devices, CA, USA). The PA standard solution used was an extract from 'CDC Acer' pea seed coats purified as described previously [41]. PA subunit composition and degree of polymerization were characterized and quantified using a method of acidcatalyzed cleavage of the PAs followed by phloroglucinol derivatization (phloroglucinolysis), as described by Jin et al. [41]. Identification of the PA subunits was confirmed by LC-MS/MS also using the method of Jin et al. [41].

\section{PA localization in pea seed coats}

Fresh seed coat tissues of 'Courier' (10, 12, 15, 20, 25, 30, and 35 DAA) located adjacent to the mid-region of the cotyledons were dissected into $1 \times 3 \mathrm{~mm}$ cross sections and immediately immerged into a fixing solution as described by Van Dongen et al. [49]. Briefly, the fixing solution consisted of $2.9 \%$ paraformaldehyde, $0.2 \%$ glutaraldehyde, $2 \mathrm{mM}$ calcium chloride $\left(\mathrm{CaCl}_{2}\right), 10 \mathrm{mM}$ sucrose, and $25 \mathrm{mM}$ piperazine-N,N'-bis(2-ethanesulfonic acid) (PIPES). The $\mathrm{pH}$ of the fixing solution was adjusted to 7.5 using sodium hydroxide. After five days of fixing solution infiltration under vacuum at room temperature, the tissues were rinsed three times with $25 \mathrm{mM}$ aqueous PIPES buffer and dehydrated using a graded ethanol series of $30 \%$ and $50 \%$ ethanol in $25 \mathrm{mM}$ PIPES buffer ( $\mathrm{pH} 7.5 ; \mathrm{v} / \mathrm{v})$, followed by $70 \%, 96 \%$, and $100 \%$ ethanol in water for 15 min each. After two more changes in 100\% ethanol followed by two changes in propylene oxide, the tissues were then submerged into 1:1 Spurr's resin and propylene oxide mixture for 2 hours. Then, the tissues were properly oriented into Spurr's resin bath and cured at $60^{\circ} \mathrm{C}$ for 3 days. Tissue sections were sliced into $4 \mu \mathrm{m}$-thick sections using a Reichert Jung Ultracut E ultra-microtome (Scotia, NY, USA), affixed onto clean slides, and placed at $60^{\circ} \mathrm{C}$ until dry. For PA localization in developing pea seed coats, a celloidin coating was applied to all slides prior to the staining process to improve the adherence of tissue sections to the glass slides. Briefly, the slides were submerged into absolute ethanol for 10 seconds, then coated with celloidin solution ( $0.5 \%$ celloidin in $1: 1$ ethanol: ethyl ether) for $5 \mathrm{~min}$, and rinsed with $70 \%$ ethanol. Tissue sections were then stained with $0.1 \%$ 4-dimethylaminocinnamaldehyde (DMACA) solution at $60^{\circ} \mathrm{C}$ for $30 \mathrm{~min}$.
The slides were washed with $100 \%$ ethanol and dehydrated with two changes of toluene for $5 \mathrm{~min}$ each. Cover slides were placed on slide-mounted tissue sections using DPX mounting media (BDH Chemicals). Tissue sections were observed using a Zeiss AXIO scope A1 light microscope (Zeiss, Germany) and micrographs were taken with a microscope-mounted Optronics camera (Optronics, CA, USA) controlled by Picture Frame ${ }^{\mathrm{TM}}$ Application 2.3 software.

\section{4-Pyrosequencing}

Total RNA was isolated from 10 to 25 DAA 'Courier' seed coats ( 4 to $5 \mathrm{~g}$ ) using an adopted CTAB (hexadecyltrimethylammonium bromide) buffer extraction method [50]. mRNA was purified from the total RNA preparation using an Oligotex mRNA Mini kit (Qiagen). Double stranded cDNA was synthesized from the mRNA according to the Joint Genome Institutes (US Department of Energy) cDNA Library Creation 454 Protocol (my.jgi.doe. gov/general/protocols/) and quantified using the Quant-iT PicoGreen dsDNA assay (Invitrogen). Approximately $2 \mu \mathrm{g}$ of cDNA was sent to the National Research Council Plant Biotechnology Institute (NRC-PBI, Saskatoon, Canada) for sequencing using a Roche 454 Titanium pyrosequencer. Transcriptome assembly was performed by personnel at the National Research Council Plant Biotechnology Institute (NRC-PBI; Saskatoon, Canada) using GS De Novo Assembler version 2.6 (Roche, Branford, Connecticut).

\section{cDNA sequence deposition}

The sequences of cDNAs described in this work were deposited in the GenBank data library under the following accession number: PsANR, KF516483; PsDFR, KF516484; PSLAR, KF516485.

\section{Additional files}

Additional file 1: Table S1. Characterization of phloroglucinolysis products from pea seeds using LC-MS-MS analysis.

Additional file 2: Figure S1. MS/MS patterns of ANR- and DFR/LARproducts. A, MS/MS data for ANR-products and cis-flavan-3-ol standards are shown. B, MS/MS data for DFR/LAR-products and tran-flavan-3-ol standards are shown.

Additional file 3: Figure S2. Incubation of recombinant PsANR with epicatechin. A, (-)-epicatechin standard (retention time; RT, $6.30 \mathrm{~min}$ ). $\mathrm{B},(+)$-catechin standard (RT, $4.85 \mathrm{~min})$. C, PsANR incubated with epicatechin (RT, $6.26 \mathrm{~min}$ ).

Additional file 4: Table S2. Top 20 unigenes in 'Courier' 10-25 DAA seed coat transcriptome. Following assembly and UniProt annotation, contigs were sorted based on the number of reads comprising each contig.

Additional file 5: Figure S3. Alignment of LAR protein sequences Pisum sativum (Ps; KF516485), Medicago truncatula (Mt; XP_003591830.1), Lotus corniculatus (LC; LAR2-1, ABC71328.1; LAR2-2, ABC71331.1), Desmodium uncinatum (Du; Q84V83.1), Phaseolus coccineus (PC; CAI56322.1), Vitis vinifera (Vv; CAl26309.1). LAR characteristic amino acid motifs RFLP, ICCN, and THD marked by black bars above the Pisum sativum sequence. 
Additional file 6: Figure S4. Expression of PSANR, PSLAR, and PSDFR in E. coli and purification of their recombinant proteins. A) Recombinant PSANR. Crude extract (lane 1), washes 1 and 2 (lanes 2 and 3, respectively) and elutent (lane 4). B) Recombinant PsDFR and PsLAR. Lanes 1,2 and 3 represent crude soluble protein, crude insoluble protein and purified concentrated protein, respectively, in PSDFR expressing culture. Lanes 4, 5 and 6 represent the similar samples from PsLAR expressing culture. Each lane contains approximately 12-15 $\mu \mathrm{g}$ of protein visualized by Coomassie staining

Additional file 7: Figure S5. RT-PCR and immunoblot analyses of PsLAR transcript and protein in Arabidopsis wild-type and Arabidopsis ANR knock-out lines. A) RT-PCR of PsLAR transcript (trans-transcript, above) and AtDFR (native transcript, below). Col: Arabidopsis Columbia; ANR KO: Arabidopsis ANR knock-out. Col GFP indicates GFP-expressing Arabidopsis Columbia line, which was used as a negative control. B) Immonoblot analysis of FLAG-tagged PsLAR (above) and Coumassie stained SDS-PAGE gels (below). C) ESI-LC-MS $\left[\mathrm{M}+\mathrm{H}^{+}(\mathrm{m} / \mathrm{z}=291)\right.$ extracted ion chromatographs from authentic (+)-catechin (left) compared to in vitro assays using crude total soluble protein extracted from PsLAR transgenic Arabidopsis siliques supplemented with (blue) or without (red) recombinant PSDFR and a vector control line with PsDFR (black).

Additional file 8: Figure S6. Analysis of seed coat color and PA chemical profile from transgenic plants. A) Dried seeds (above) and p-dimethylaminocinnamaldehyde (DMACA) stained seeds (below) from GFP vector control line and ANR KO-PsLAR transgenic lines show a lack of proanthocyanidins or flavan-3-ols in the seed coat. B) HPLC chromatograms of the phloroglucinol acid hydrolysis products of proanthocyanidins extracted from the mature seeds of Col PSLAR 5 transgenic lines. No catechin-phloroglucinol (PA extension units; solid arrow) or catechin (PA terminal units; dashed arrow) were detected in Col LAR5. 'LAN3017' seed coat proanthocyanidins were used as a control for catechin-phloroglucinol. Peak 1, catechin-phloroglucinol; Peak 2, epicatechinphloroglucinol; Peak 3 and peak 4 are (+)-catechin and (-)-epicatechin standards, respectively

Additional file 9: Table S3. List of PCR primers used in this study

\section{Abbreviations}

PA: Proanthocyanidin; mDP: Mean degree of polymerization; DAA: Days after anthesis; EC: Epicatechin; EAZ: Epiafzelechin; EGC: Epigallocatechin; DHM: Dihydromyricetin; DHQ: Dihydroquercetin; CT: Catechin GC: Gallocatechin; AZ: Afzelechin; DMACA: p-dimethyl aminocinnamaldehyde; EST: Expressed sequence tag.

\section{Competing interests}

The authors declare that they have no competing interests.

\section{Authors' contributions}

$\mathrm{KF}$ carried out biochemical and molecular analyses (transcriptomics, recombinant enzyme studies, GRT-PCR, and transgenic analyses), and provided the initial manuscript draft. AJ grew and harvested the pea seed coat tissues for all analyses in this study, isolated and chemically characterized the pea PAs, performed the histological studies, and drafted the text for the histological and chemical analyses. TDN performed enzyme assays using the protein extract of Arabidopsis siliques. DKR, DMR, and JAO designed experiments, interpreted the results, and revised the manuscript. The Ozga-Reinecke and Ro labs contributed equally to the work presented in this manuscript. All authors read and approved the final manuscript.

\section{Acknowledgements}

We thank Jacek Nowak and Dustin Cram at the National Research Council Plant Biotechnology Institute (NRC-PBI, Canada) for their expert support in 454-sequencing data processing and analysis. This work was supported by the Natural Sciences and Engineering Research Council of Canada (NSERC) and Alberta Innovates Bio Solutions (Al Bio) to J.A. Ozga and D.K. Ro. This work was also supported by the Next-Generation BioGreen 21 Program (SSAC grant PJ009549032014), Rural Development Administration, Republic of Korea to D.K. Ro.

\section{Author details}

'Department of Biological Sciences, University of Calgary, 2500 University Dr. NW, Calgary, Alberta, Canada. ${ }^{2}$ Plant BioSystems, Department of Agricultural,
Food, and Nutritional Science, University of Alberta, Edmonton, Alberta, Canada.

Received: 17 August 2014 Accepted: 2 September 2014 Published: 16 September 2014

\section{References}

1. Guillon F, Champ MM-J: Carbohydrate fractions of legumes: uses in human nutrition and potential for health. Br J Nutr 2002, 88:293-306.

2. Lepiniec L, Debeaujon I, Routaboul JM, Baudry A, Pourcel L, Nesi N, Caboche M: Genetics and biochemistry of seed flavonoids. Annu Rev Plant Biol 2006, 57:405-430.

3. Dixon RA, Xie DY, Sharma SB: Proanthocyanidins-a final frontier in flavonoid research? New Phytol 2005, 165:9-28.

4. Rasmussen SE, Frederiksen H, Struntze Krogholm K, Poulsen L: Dietary proanthocyanidins: occurrence, dietary intake, bioavailability, and protection against cardiovascular disease. Mol Nutr Food Res 2005, 49:159-174.

5. Lee YA, Cho EJ, Yokozawa T: Effects of proanthocyanidin preparations on hyperlipidemia and other biomarkers in mouse model of type 2 diabetes. J Agric Food Chem 2008, 56:7781-7789.

6. Manach C, Williamson G, Morand C, Scalbert A, Rémésy C: Bioavailability and bioefficacy of polyphenols in humans. I. Review of 97 bioavailability studies. Am J Clin Nutr 2005, 81:230S-242S.

7. Holton TA, Brugliera F, Lester DR, Tanaka Y, Hyland CD, Menting JGT, Lu C-Y, Farcy E, Stevenson TW, Cornish EC: Cloning and expression of cytochrome P450 genes controlling flower colour. Nature 1993, 366:276-279.

8. Brugliera F, Barri-Rewell G, Holton TA, Mason JG: Isolation and characterization of a flavonoid 3'-hydroxylase CDNA clone corresponding to the Ht1 locus of Petunia hybrida. Plant J 1999, 19:441-451.

9. Britsch L, Grisebach H: Purification and characterization of (2S)-flavanone 3-hydroxylase from Petunia hybrida. Eur J Biochem 1986, 156:569-577.

10. Reddy AR, Britsch L, Salamini F, Saedler H, Rohde W: The A1 (anthocyanin1) locus in Zea mays encodes dihydroquercetin reductase. Plant Sci 1987, 52:7-13.

11. Pelletier MK, Murrell JR, Shirley BW: Characterization of flavonol synthase and leucoanthocyanidin dioxygenase genes in Arabidopsis (further evidence for differential regulation of "early" and "late" genes). Plant Physiol 1997, 113:1437-1445.

12. Xie DY, Sharma SB, Paiva NL, Ferreira D, Dixon RA: Role of anthocyanidin reductase, encoded by BANYULS in plant flavonoid biosynthesis. Science 2003, 299:396-399.

13. Tanner GJ, Francki KT, Abrahams S, Watson JM, Larkin PJ, Ashton AR: Proanthocyanidin biosynthesis in plants. Purification of legume leucoanthocyanidin reductase and molecular cloning of its CDNA. J Biol Chem 2003, 278:31647-31656.

14. Abrahams S, Lee E, Walker AR, Tanner GJ, Larkin PJ, Ashton AR: The Arabidopsis TDS4 gene encodes leucoanthocyanidin dioxygenase (LDOX) and is essential for proanthocyanidin synthesis and vacuole development. Plant J 2003, 35:624-636.

15. Kitamura S, Shikazono N, Tanaka A: TRANSPARENT TESTA 19 is involved in the accumulation of both anthocyanins and proanthocyanidins in Arabidopsis. Plant J 2004, 37:104-114.

16. Marinova K, Pourcel L, Weder B, Schwarz M, Barron D, Routaboul JM, Debeaujon I, Klein M: The Arabidopsis MATE transporter TT12 acts as a vacuolar flavonoid/H + -antiporter active in proanthocyanidinaccumulating cells of the seed coat. Plant Cell 2007, 19:2023-2038.

17. Zhao J, Dixon RA: MATE transporters facilitate vacuolar uptake of epicatechin 3'-O-glucoside for proanthocyanidin biosynthesis in Medicago truncatula and Arabidopsis. Plant Cell 2009, 21:2323-2340.

18. Pang $Y$, Peel GJ, Sharma SB, Tang Y, Dixon RA: A transcript profiling approach reveals an epicatechin-specific glucosyltransferase expressed in the seed coat of Medicago truncatula. Proc Natl Acad Sci U S A 2008, 105:14210-14215.

19. Baxter IR, Young JC, Armstrong G, Foster N, Bogenschutz N, Cordova T, Peer WA, Hazen SP, Murphy AS, Harper JF: A plasma membrane $\mathrm{H}^{+}$-ATPase is required for the formation of proanthocyanidins in the seed coat endothelium of Arabidopsis thaliana. Proc Natl Acad Sci U S A 2005, 102:2649-2654.

20. Kitamura S, Matsuda F, Tohge T, Yonekura-Sakakibara K, Yamazaki M, Saito K, Narumi I: Metabolic profiling and cytological analysis of 
proanthocyanidins in immature seeds of Arabidopsis thaliana flavonoid accumulation mutants. Plant J 2010, 62:549-559.

21. Mueller LA, Goodman CD, Silady RA, Walbot V: AN9, a petunia glutathione $\mathrm{S}$-transferase required for anthocyanin sequestration, is a flavonoidbinding protein. Plant Physiol 2000, 123:1561-1570.

22. Poustka F, Irani NG, Feller A, Lu Y, Pourcel L, Frame K, Grotewold E: A trafficking pathway for anthocyanins overlaps with the endoplasmic reticulum-to-vacuole protein-sorting route in Arabidopsis and contributes to the formation of vacuolar inclusions. Plant Physiol 2007, 145:1323-1335.

23. Brillouet J-M, Romieu C, Schoefs B, Solymosi K, Cheynier V, Fulcrand H, Verdeil $\mathrm{J}-\mathrm{L}$, Conéjéro $\mathrm{G}$ : The tannosome is an organelle forming condensed tannins in the chlorophyllous organs of Tracheophyta. Ann Bot 2013, 112:1003-1014.

24. Pourcel L, Routaboul J-M, Kerhoas L, Caboche M, Lepiniec L, Debeaujon I: TRANSPARENT TESTA10 encodes a laccase-like enzyme involved in oxidative polymerization of flavonoids in Arabidopsis seed coat. Plant Cell 2005, 17:2966-2980

25. Davin LB, Wang H-B, Crowell AL, Bedgar DL, Martin DM, Sarkanen S, Lewis NG: Stereoselective bimolecular phenoxy radical coupling by an auxiliary (dirigent) protein without an active center. Science 1997, 275:362-367.

26. Pang Y, Peel GJ, Wright E, Wang Z, Dixon RA: Early steps in proanthocyanidin biosynthesis in the model legume Medicago truncatula. Plant Physiol 2007, 145:601-615.

27. Debeaujon I, Nesi N, Perez P, Devic M, Grandjean O, Caboche M, Lepiniec L. Proanthocyanidin-accumulating cells in Arabidopsis testa: regulation of differentiation and role in seed development. Plant Cell 2003, 15:2514-2531.

28. Smýkal P, Aubert G, Burstin J, Coyne CJ, Ellis NTH, Flavell AJ, Ford R, Hýbl M, Macas J, Neumann P, McPhee KE, Redden RJ, Rubiales D, Weller JL, Warkentin TD: Pea (Pisum sativum L.) in the Genomic Era. Agronomy 2012, 2:74-115.

29. Gutmann M, Feucht W: A new method for selective localization of flavan-3-ols in plant tissues involving glycolmethacrylate embedding and microwave irradiation. Histochemistry 1991, 96:83-86.

30. Kennedy JA, Jones GP: Analysis of proanthocyanidin cleavage products following acid-catalysis in the presence of excess phloroglucinol. J Agric Food Chem 2001, 49:1740-1746.

31. Porter $L$, Hrstich LN, Chan BG: The conversion of procyanidins and prodelphinidins to cyanidin and delphinidin. Phytochemistry 1985, 25:223-230.

32. Gargouri M, Chaudière J, Manigand C, Mauge C, Bathany K, Schmitter J-M, Gallois B: The epimerase activity of anthocyanidin reductase from Vitis vinifera and its regiospecific hydride transfers. Biol Chem 2010, 391:219-227.

33. Pang Y, Abeysinghe ISB, He J, He X, Huhman D, Mewan KM, Sumner LW, Yun J, Dixon RA: Functional characterization of proanthocyanidin pathway enzymes from tea and their application for metabolic engineering. Plant Physiol 2013, 161:1103-1116.

34. Franssen S, Shrestha R, Bräutigam A, Bornberg-Bauer E, Weber A: Comprehensive transcriptome analysis of the highly complex Pisum sativum genome using next generation sequencing. BMC Genomics 2011, 11:227-242.

35. Kaur S, Pembleton LW, Cogan NOI, Savin KW, Leonforte T, Paull J, Materne $M$, Forster JW: Transcriptome sequencing of field pea and faba bean for discovery and validation of SSR genetic markers. BMC Genomics 2012, 13:104-115.

36. Chevreux B, Wetter T, Suhai S: Genome sequence assembly using trace signals and additional sequence information. In German Conference on Bioinformatics; Hannover, Germany. 1999:45-56.

37. Nadeau CD, Ozga JA, Kurepin LV, Jin A, Pharis RP, Reinecke DM: Tissue-specific regulation of gibberellin biosynthesis in developing pea seeds. Plant Physiol 2011, 156:897-912

38. Sreenivasulu N, Wobus U: Seed-development programs: a systems biology-based comparison between dicots and monocots. Annu Rev Plant Biol 2013, 64:189-217.

39. Bogs J, Downey MO, Harvey JS, Ashton AR, Tanner GJ, Robinson SP: Proanthocyanidin synthesis and expression of genes encoding leucoanthocyanidin reductase and anthocyanidin reductase in developing grape berries and grapevine leaves. Plant Physiol 2005, 139:652-663.

40. Gu LW, Kelm MA, Hammerstone JF, Beecher G, Holden J, Haytowitz D, Gebhardt S, Prior RL: Concentrations of proanthocyanidins in common foods and estimations of normal consumption. J Nutr 2004, 134:613-617.
41. Jin A, Ozga JA, Lopes-Lutz D, Schieber A, Reinecke DM: Characterization of proanthocyanidins in pea (Pisum sativum L.), lentil (Lens culinaris L.), and faba bean (Vicia faba L.) seeds. Food Res Int 2012, 46:528-535.

42. Lin Y-L, Juan IM, Chen Y-L, Liang Y-C, Lin J-K: Composition of polyphenols in fresh tea leaves and associations of their oxygen-radical-absorbing capacity with antiproliferative actions in fibroblast cells. J Agric Food Chem 1996, 44:1387-1394.

43. Rice-Evans C, Miller N, Paganga G: Antioxidant properties of phenolic compounds. Trends Plant Sci 1997, 2:152-159.

44. Singh S, McCallum J, Gruber MY, Towers GHN, Muir AD, Bohm BA, KoupaiAbyazani MR, Glass ADM: Biosynthesis of flavan-3-ols by leaf extracts of Onobrychis viciifolia. Phytochemistry 1997, 44:425-432.

45. Wang $S X$, Hunter W, Plant A: Isolation and purification of functional total RNA from woody branches and needles of sitka and white spruce. Biotechniques 2000, 28:292-296.

46. Karimi M, Inzé D, Depicker A: GATEWAY ${ }^{\mathrm{TM}}$ vectors for Agrobacterium-mediated plant transformation. Trends in Plant Sci 2002, 7:193-195.

47. Zhang X, Henriques R, Lin S-S, Niu Q-W, Chua N-H: Agrobacterium-mediated transformation of Arabidopsis thaliana using the floral dip method. Nat Protoc 2006, 1:641-646.

48. Pfaff MW: A new mathematical model for relative quantification in real-time RT-PCR. Nucleic Acids Res 2001, 29:e45.

49. Van Dongen JT, Ammerlaan AMH, Wouterlood M, Van Aelst AC, Borstlap AC: Structure of the developing pea seed coat and the post-phloem transport pathway of nutrients. Ann Bot 2003, 91:729-737.

50. Meisel L, Fonseca B, González S, Baeza-Yates R, Cambiazo V, Campos R, Gonzalez M, Orellana A, Retamales J, Silva H: A rapid and efficient method for purifying high quality total RNA from peaches (Prunus persica) for functional genomics analyses. Biol Res 2005, 38:83-88.

doi:10.1186/s12870-014-0238-y

Cite this article as: Ferraro et al: Characterization of proanthocyanidin

metabolism in pea (Pisum sativum) seeds. BMC Plant Biology 2014 14:238.

\section{Submit your next manuscript to BioMed Central and take full advantage of:}

- Convenient online submission

- Thorough peer review

- No space constraints or color figure charges

- Immediate publication on acceptance

- Inclusion in PubMed, CAS, Scopus and Google Scholar

- Research which is freely available for redistribution 